\title{
Solution to the stochastic Schrödinger equation on the full space
}

\author{
Arnaud Debussche ${ }^{1,3}$ and Jörg Martin ${ }^{2,4}$ (I) \\ ${ }^{1}$ Université de Rennes, CNRS, IRMAR-UMR 6625, F-35000 Rennes, France \\ ${ }^{2}$ Humboldt-Universität zu Berlin, Berlin, Germany \\ E-mail: arnaud.debussche@ens-rennes.fr
}

Received 20 July 2017, revised 21 November 2018

Accepted for publication 30 November 2018

Published 4 March 2019

Recommended by Dr Jean-Claude Saut

\begin{abstract}
We show here how the methods recently applied in Debussche and Weber (2018 Electron. J. Probab. 23 28) to solve the stochastic nonlinear Schrödinger equation on $\mathbb{T}^{2}$ can be enhanced to yield solutions on $\mathbb{R}^{2}$ if the nonlinearity is weak enough. We prove that the solutions remain localized on compact time intervals which allow us to apply energy methods on the full space.
\end{abstract}

Keywords: nonlinear Schrödinger equation, localization, white noise, renormalization

Mathematics Subject Classification numbers: 60H15, 35Q55

\section{Introduction}

We study the following Cauchy problem on $[0, T] \times \mathbb{R}^{2}$

$$
\imath \partial_{t} u=\Delta u+\lambda u|u|^{2 \sigma}+u \xi, u(0)=u_{0},
$$

where $\sigma>0, \lambda \in \mathbb{R}$ are parameters and $\xi \in \mathcal{S}^{\prime}\left(\mathbb{R}^{2}\right)$ stands for white noise in space. The unknown $u$ is a complex valued process. Equation (1) can be seen as a stochastic version of the well-studied deterministic nonlinear Schrödinger equation (for example [BG80, Caz79, Caz03, Kat87]), where the noise term $u \xi$ is absent. The case $\sigma<1$ is known as the sub-critical regime and $\sigma \geqslant 1$ as (super-)critical. For a positive coefficient $\lambda>0$ the equation is called focusing, while for $\lambda<0$ one uses the term defocusing. For a focusing (super-)critical nonlinearity, solutions may form singularity in finite time in the deterministic case.

\footnotetext{
${ }^{3}$ Partially supported by the French government thanks to the 'Investissements d'Avenir' program ANR-11LABX-0020-01.

${ }^{4}$ Financial support by the DFG via Research Training Group RTG 1845 is gratefully acknowledged. 
While the deterministic equation arises in nonlinear optics to model laser propagation in a dispersive material [Ber98, section 1.1.-1.3.], the stochastic term can be seen as taking into account disorder in the considered medium. In the physical context interesting effects such as Anderson localization and the existence of solitons have been observed [And58, Con12, FC10].

Equation (1) with $\sigma=1$ on the two dimensional torus $\mathbb{T}^{2}$ was recently studied in [DW18]. However, most of physical phenomena described by (1) are set on the full space $\mathbb{R}^{2}$. For instance, if one is interested in solitary waves and their stability, it is important to have a notion for a solution on $\mathbb{R}^{2}$. This question is the main motivation for this work which is concerned with wellposedness for (1). Qualitative properties of the solutions will be the object of further studies.

Due to the irregularity of the white noise, the product $u \xi$ is not well defined and a renormalization is necessary. The situation is in fact similar to the parabolic Anderson model studied in [HL15] where a change of unknown is introduced. The same transformation is used in [DW18] and global existence of solutions for a renormalized equation on $\mathbb{T}^{2}$ is obtained.

In [DW18] the transformation $v:=\mathrm{e}^{Y} u$, where $Y$ solves $\Delta Y=\xi$, is introduced. This converts (1) into a new equation which turns out to be better behaved:

$$
\imath \partial_{t} v=\Delta v+v \nabla Y^{2}-2 \nabla v \nabla Y+\lambda|v|^{2 \sigma} v \mathrm{e}^{-2 \sigma Y}, v(0)=v_{0}:=\mathrm{e}^{Y} u_{0} .
$$

Alas, similar as in [DW18] (and [HL15]), the term $\nabla Y^{2}$ is ill-defined as a square of a distribution, but can be replaced by a meaningful object $: \nabla Y^{2}$ :, which is essentially the Wick product of $\nabla Y$ with itself, so that the following equation is considered instead

$$
\imath \partial_{t} v=\Delta v+v: \nabla Y^{2}:-2 \nabla v \nabla Y+\lambda|v|^{2 \sigma} v \mathrm{e}^{-2 \sigma Y}, v(0)=v_{0}:=\mathrm{e}^{Y} u_{0} .
$$

The replacement $\nabla Y^{2} \rightarrow: \nabla Y^{2}$ : corresponds to the subtraction of a renormalization term ' $-\infty \cdot u$ ' in the original relation (1) or, equivalently, to the cancellation of a singular phase factor ' $\mathrm{e}$ ¿

We proceed similarly here with some modifications. First, as in [HL15], $Y$ does not solve exactly the equation $\Delta Y=\xi$. A truncated Green function is used and in fact $Y$ satisfies $\Delta Y=\xi+\varphi * \xi$ for a smooth and compactly supported $\varphi$. The renormalization step defining $: \nabla Y^{2}$ : as well as other properties of $Y$ are described in section 2.3 and the modified equation, similar to (2), is stated there, see (27).

A key observation in [DW18] is that the energy

$\int_{\mathbb{R}^{2}}\left(\frac{1}{2}|\nabla v(t, x)|^{2}-\frac{1}{2}|v(t, x)|^{2}: \nabla Y^{2}:(x)-\frac{\lambda}{2 \sigma+2}|v(t, x)|^{2 \sigma+2} \mathrm{e}^{-2 \sigma Y(x)}\right) \mathrm{e}^{-2 Y(x)} \mathrm{d} x$

is a conserved quantity, i.e. independent of the time $t$. It is shown that all terms make sense and this fact was used in order to derive an $H^{1}$ bound on $v$ for (2) on a torus.

On a full space set-up such an approach is more delicate as the white noise grows for $x \rightarrow \infty$ essentially like $\sqrt{\log (|x|)}$ (compare lemma 2.3 below) and we thus need control over the decay of $v$ to make sense of the integration in (3) over the full space. This is achieved by lemma 3.1 below which states that almost surely (a.s.)

$$
\sup _{t \in[0, T]} \int_{\mathbb{R}^{2}}|x|^{2 \delta} \cdot|v(t, x)|^{2} \mathrm{~d} x<\infty
$$

for $\delta>0$ small enough, provided we have some control over the gradient $\nabla v$ and that the initial condition satisfies a.s. $v_{0} \in H^{1}$ and

$$
\int_{\mathbb{R}^{2}}|x|^{2 \delta_{0}}\left(\left|v_{0}(x)\right|^{2}+\left|\nabla v_{0}(x)\right|^{2}\right) \mathrm{d} x<\infty
$$


for some $\delta_{0}>\delta$. The localization property (4) allows us to make sense of (3) and to derive bounds on $v$ in adequate weighted Sobolev spaces.

In order to construct a solution to (2) we consider an approximation $v_{\varepsilon}$ which solves

$$
\imath \partial_{t} v_{\varepsilon}=\Delta v_{\varepsilon}+v_{\varepsilon}: \widetilde{\nabla Y_{\varepsilon}^{2}}:-2 \nabla v_{\varepsilon} \nabla Y_{\varepsilon}+\lambda\left|v_{\varepsilon} \mathrm{e}^{-Y_{\varepsilon}}\right|^{2 \sigma} v_{\varepsilon}, v_{\varepsilon}(0)=v_{0}
$$

with $\widetilde{\nabla Y_{\varepsilon}^{2}}:=: \nabla Y_{\varepsilon}^{2}:-\varphi * \xi_{\varepsilon}$ (see section 2.3 for the precise definition). We take the same initial condition but the equation is driven by a $Y_{\varepsilon}$ that satisfies $\Delta Y_{\varepsilon}=\xi_{\varepsilon}+\varphi * \xi_{\varepsilon}$ where $\xi_{\varepsilon}=\varepsilon^{-2} \rho\left(\varepsilon^{-1} \cdot\right) * \xi$ is a mollification of the considered noise. We use (3) and (4) for this regularized solution. Then under the additional assumption

$$
\int_{\mathbb{R}^{2}}|x|^{2 \delta_{0}}\left(\left|v_{0}(x)\right|^{2}+\left|\nabla v_{0}(x)\right|^{2}+\left|\nabla^{2} v_{0}(x)\right|^{2}\right) \mathrm{d} x<\infty
$$

we reproduce the arguments from [DW18] to derive a bound in a weighted $H^{2}$ space which grows as a power of $\ln \varepsilon$ and then show that the sequence obtained by taking $\varepsilon=2^{-k}$ is a.s. Cauchy in the space of continuous functions from positive time to a weighted $H^{\gamma}$ space, $\gamma<2$. Unfortunately, for these two steps we have to impose restrictions on the nonlinear term. We assume that either the equation is defocusing $\lambda \leqslant 0$ or $\sigma<1$ and prove local existence and uniqueness of a solution of (1) once renormalized and transformed as above, see (27) below. The initial data is assumed to satisfy (6) for some $\delta_{0}>0$. Then under the additional assumption $\sigma<1 / 2$, we prove that the solution is in fact global in time. For a more precise formulation of our results, see theorems 4.4 and 5.2 below.

It might seem surprising that even for local well-posedness the sign of $\lambda$ matters. Recall that in the absence of noise, local well-posedness is known in the energy space for any $\sigma \geqslant 0$ in dimension 2 thanks to the Strichartz estimates. We do not know whether similar estimates hold for the noisy equation considered here and have to work as in [DW18].

Also, in [DW18], the restriction on the nonlinear term is similar to the deterministic case for global existence: $\sigma<1$. A key ingredient is the use of Brezis-Galouet inequality. In the present article, this inequality is not easy to use since it is not adapted to weighted spaces. We have to rely on new arguments and use complicated estimates to get both the local and the global existence results.

Our analysis will be formulated in the language of weighted Besov spaces whose definition and fundamental properties we recall in section 2 below. We then describe the growth behavior of the noise in our equation in this framework and finally give a rigorous formulation of the problem we solve. Roughly speaking, section 3 is devoted to the control of moments and of the energy, while sections 4 and 5 show an $H^{\gamma}$ bound for $\gamma \in(1,2)$ which allows for a solution of (1) in theorems 4.4 and 5.2.

\section{Notation}

We write $c>0$ or $C>0$ for deterministic constants and $K_{\varepsilon}$ for random constants depending on $\varepsilon \in(0,1]$ with $L^{p}(\mathbb{P})$ norms bounded independently of $\varepsilon$ for $p \in[1, \infty)$ on the considered probability space with measure $\mathbb{P}$ :

$$
\mathbb{E}\left(K_{\varepsilon}^{p}\right) \leqslant c_{p},
$$

for any $p \geqslant 1$ where $c_{p}$ is a constant depending on $p$ but not on $\varepsilon$ and $\mathbb{E}$ is the expectation associated to $\mathbb{P}$.

We also write $K_{k}=K_{2^{-k-1}} K_{2^{-k}}$ and indicate further by this notation that this sequence is bounded almost surely in $k=0,1,2, \ldots$ We sometimes use $a>0$ for a positive, deterministic 
constant appearing in an exponent. The constants $c, C, K_{\varepsilon}, K_{k}$ and $a$ may change from one line to another.

An important role in this paper will be played by the polynomial weights

$$
\langle x\rangle^{\mu}:=\left(1+|x|^{2}\right)^{\mu / 2},
$$

with $\mu \in \mathbb{R}$. For weighted $L^{p}$ spaces $L^{p}\left(\mathbb{R}^{d},\langle x\rangle^{\mu}\right)=L^{p}\left(\langle x\rangle^{\mu}\right), p \in[1, \infty], \mu \in \mathbb{R}$ we here use the convention

$$
\|f\|_{L^{p}\left(\langle x\rangle^{\mu}\right)}:=\left\|\langle x\rangle^{\mu} f\right\|_{L^{p}}=\left(\int_{\mathbb{R}^{d}}\left|f(x)\langle x\rangle^{\mu}\right|^{p} \mathrm{~d} x\right)^{1 / p},
$$

(with the usual interpretation if $p=\infty$ ). If $\mu=0$ we simply write $L^{p}=L^{p}\left(\langle x\rangle^{0}\right)$.

We will sometimes use formal notations for distributions $f \in \mathcal{S}^{\prime}\left(\mathbb{R}^{d}\right)$ such as

$$
\int_{\mathbb{R}^{d}} f(x) \phi(x) \mathrm{d} x:=f(\phi)
$$

for $\phi \in \mathcal{S}\left(\mathbb{R}^{d}\right)$.

Unless explicitly stated, the letter $T>0$ denotes a deterministic time horizon. The letter $\tau>0$ will be used for random times. Given a normed space $X$ we write $C_{T} X$ for the space of continuous functions $f:[0, T] \rightarrow X$ and introduce

$$
\|f\|_{C_{T} X}:=\sup _{t \in[0, T]}\|f(t)\|_{X}
$$

Given a function $f:[0, T] \times \mathbb{R}^{d} \rightarrow \mathbb{C}$ which depends on time and space we will write for any $t \in[0, T]$ the symbol $f(t)$ to denote the function $f(t): \mathbb{R}^{d} \ni x \mapsto f(t, x) \in \mathbb{C}$. Most of the further notations and used function spaces are described in section 2.1.

\section{Definitions and basics}

\subsection{Weighted Besov and Sobolev spaces}

We follow here closely [Tri06] and [BCD11] and refer to these books for details. The definition of weighted Besov spaces on $\mathbb{R}^{d}$ used in this article is

$\mathcal{B}_{p, q}^{\alpha}\left(\mathbb{R}^{d},\langle x\rangle^{\mu}\right)=\mathcal{B}_{p, q}^{\alpha}\left(\langle x\rangle^{\mu}\right)=\left\{f \in \mathcal{S}^{\prime}\left(\mathbb{R}^{d}\right) \mid\|f\|_{\mathcal{B}_{p, q}^{\alpha}\left(\langle x\rangle^{\mu}\right)}:=\left\|2^{j \alpha}\right\| \Delta_{j} f\left\|_{L^{p}\left(\langle x\rangle^{\mu}\right)}\right\|_{\ell^{q}}<\infty\right\}$,

where $\left(\Delta_{j}\right)_{j=-1,0, \ldots}$ denotes some choice of Littlewood-Paley-blocks and where $p, q \in[1, \infty]$ and $\alpha, \mu \in \mathbb{R}$. We will write $\mathcal{B}_{p, q}^{\alpha}=\mathcal{B}_{p, q}^{\alpha}\left(\mathbb{R}^{d}\right)$ for the unweighted space $\mathcal{B}_{p, q}^{\alpha}\left(\langle x\rangle^{0}\right)$. We will sometimes write for $p, q \in[1, \infty], \alpha, \mu \in \mathbb{R}$

$$
\mathcal{B}_{p, q}^{\alpha^{-}}\left(\langle x\rangle^{\mu}\right):=\bigcap_{\alpha^{\prime}<\alpha} \mathcal{B}_{p, q}^{\alpha^{\prime}}\left(\langle x\rangle^{\mu}\right)
$$

A convenient property of the spaces in (7) is that the weight can be "pulled in",

$$
\|f\|_{\mathcal{B}_{p, q}^{\alpha}\left(\langle x\rangle^{\mu}\right)} \approx\left\|f\langle x\rangle^{\mu}\right\|_{\mathcal{B}_{p, q}^{\alpha}}
$$

in the sense of equivalent norms, for $\alpha, \mu \in \mathbb{R}$ and $p, q \in[1, \infty]$, compare [Tri06, theorem 6.5]. Relation (8) can be used to translate results from the unweighted spaces to their weighted analogues. Let us recall a few useful properties of the spaces $\mathcal{B}_{p, q}^{\alpha}\left(\langle x\rangle^{\mu}\right)$. 
Lemma 2.1. The weighted Besov spaces defined above have the following properties:

(i) (Besov embedding) For $\alpha_{1}, \alpha_{2}, a_{1}, a_{2} \in \mathbb{R}$ and $p_{1}, p_{2}, q_{1}, q_{2} \in[1, \infty]$ with $a_{1} \leqslant a_{2}$, $\alpha_{1}-\frac{d}{p_{1}} \leqslant \alpha_{2}-\frac{d}{p_{2}}, q_{2} \leqslant q_{1}$ and $p_{2} \leqslant p_{1}$, we have the continuous embedding

$$
\mathcal{B}_{p_{2}, q_{2}}^{\alpha_{2}}\left(\mathbb{R}^{d},\langle x\rangle^{a_{2}}\right) \subseteq \mathcal{B}_{p_{1}, q_{1}}^{\alpha_{1}}\left(\mathbb{R}^{d},\langle x\rangle^{a_{1}}\right) \text {. }
$$

(ii) (Sobolev embedding) For $\alpha>0, p \in[2, \infty]$ such that $-\frac{d}{p} \leqslant \alpha-\frac{d}{2}$ and $\mu_{1}, \mu_{2} \in \mathbb{R}, \mu_{1} \leqslant \mu_{2}$ we have the continuous embedding

$$
\mathcal{B}_{2,2}^{\alpha}\left(\mathbb{R}^{d},\langle x\rangle^{\mu_{2}}\right) \subseteq L^{p}\left(\mathbb{R}^{d},\langle x\rangle^{\mu_{1}}\right) .
$$

(iii) (Duality) For $\alpha \in \mathbb{R}, p, q \in[1, \infty), \mu \in \mathbb{R}$ we have the duality

$$
\left(\mathcal{B}_{p, q}^{\alpha}\left(\langle x\rangle^{\mu}\right)\right)^{\prime}=\mathcal{B}_{p^{\prime}, q^{\prime}}^{-\alpha}\left(\langle x\rangle^{-\mu}\right),
$$

where $\frac{1}{p}+\frac{1}{p^{\prime}}=1$ and $\frac{1}{q}+\frac{1}{q^{\prime}}=1$.

(iv) (Multiplication) For $\mu_{1}, \mu_{2} \in \mathbb{R}, p_{1}, p_{2} \in[1, \infty]$ and $\alpha_{1}, \alpha_{2} \in \mathbb{R}$ with $\alpha_{1}+\alpha_{2}>0$ we have for $\alpha=\alpha_{1} \wedge \alpha_{2} \wedge\left(\alpha_{1}+\alpha_{2}\right), \frac{1}{p}=\frac{1}{p_{1}}+\frac{1}{p_{2}}, \tilde{p}=p_{1} \vee p_{2}$ and $\mu=\mu_{1}+\mu_{2}$

$$
\left\|f_{1} \cdot f_{2}\right\|_{\mathcal{B}_{p, \tilde{p}}^{\alpha}\left\langle\langle x\rangle^{\mu}\right)} \leqslant C\left\|f_{1}\right\|_{\mathcal{B}_{p_{1}, p_{1}}^{\alpha_{1}}\left(\langle x\rangle^{\mu}\right)}\left\|f_{2}\right\|_{\mathcal{B}_{p_{2}, p_{2}}^{\alpha_{2}}\left(\langle x\rangle^{\mu_{2}}\right)}
$$

and for any $\kappa>0$

$$
\left\|f_{1} \cdot f_{2}\right\|_{\mathcal{B}_{p, p}^{\alpha-\kappa}\left(\langle x\rangle^{\mu}\right)} \leqslant C\left\|f_{1}\right\|_{\mathcal{B}_{p_{1}, p_{1}}^{\alpha_{1}}\left(\langle x\rangle^{\mu_{1}}\right)}\left\|f_{2}\right\|_{\mathcal{B}_{p_{2}, p_{2}}^{\alpha_{2}}\left(\langle x\rangle^{\mu_{2}}\right)} .
$$

(v) (Mollification) Given a $\rho \in L^{1}$ with compact support, $\alpha \in \mathbb{R}, p, q \in[1, \infty], \mu \in \mathbb{R}$ and $\varepsilon \in(0,1]$ we have with $\rho_{\varepsilon}=\varepsilon^{-d} \rho\left(\varepsilon^{-1}\right)$

$$
\left\|\rho_{\varepsilon} * f\right\|_{\mathcal{B}_{p, q}^{\alpha}\left(\langle x\rangle^{\mu}\right)} \leqslant C\|f\|_{\mathcal{B}_{p, q}^{\alpha}\left(\langle x\rangle^{\mu}\right)} .
$$

Proof. Property (iv) is a special case of the far more general paraproduct inequalities. We refer to [BCD11] or, for a slightly more general version (which was applied here), to [PT16]. Property (i) and (iii) are standard [Tri83, theorems 2.7.1 and 2.11.2], property (ii) is probably also standard but is also a direct consequence of [Tri06, theorems 6.7 and 6.9]. It remains to show property $(v)$. The Littlewood-Paley-blocks in the norm of (7) satisfy $\Delta_{j}\left(\rho_{\varepsilon} * f\right)=\rho_{\varepsilon} * \Delta_{j} f$ so that due to $\langle x\rangle^{\mu} \leqslant C\langle x-y\rangle^{|\mu|} \cdot\langle y\rangle^{\mu}$ we obtain

$$
\left|\langle x\rangle^{\mu} \Delta_{j}\left(\rho_{\varepsilon} * f\right)(x)\right| \leqslant C \int_{\mathbb{R}^{d}}\langle x-y\rangle^{|\mu|}\left|\rho_{\varepsilon}(x-y)\right| \cdot\langle y\rangle^{\mu}\left|\Delta_{j} f(y)\right| \mathrm{d} y .
$$

Property $(v)$ then follows from Young's convolution inequality and $\sup _{\varepsilon}\left\|\langle x\rangle^{|\mu|} \rho_{\varepsilon}\right\|_{L^{1}}<\infty$.

For $\alpha>0$ the space $\mathcal{B}_{p, q}^{\alpha}\left(\langle x\rangle^{\mu}\right)$ contains only functions. For non-positive $\alpha$ there might be genuine distributions contained in this space. We will frequently use the following identities for $\alpha, \mu \in \mathbb{R}$

$$
\begin{aligned}
\mathcal{B}_{2,2}^{\alpha}\left(\langle x\rangle^{\mu}\right) & =H^{\alpha}\left(\langle x\rangle^{\mu}\right), \\
\mathcal{B}_{\infty, \infty}^{\alpha}\left(\langle x\rangle^{\mu}\right) & =\mathcal{C}^{\alpha}\left(\langle x\rangle^{\mu}\right),
\end{aligned}
$$

where $H^{\alpha}\left(\langle x\rangle^{\mu}\right)$ is the (weighted) Bessel-potential space with the equivalent norm 


$$
\|f\|_{H^{\alpha}\left(\langle x\rangle^{\mu}\right)} \approx\left\|\mathcal{F}^{-1}\langle\cdot\rangle^{\alpha} \mathcal{F} f\right\|_{L^{2}\left(\langle x\rangle^{\mu}\right)}
$$

and where $\mathcal{C}^{\alpha}\left(\langle x\rangle^{\mu}\right)$ is the (weighted) Hölder-Zygmund space that coincides with the classical Hölder space for $\alpha \in \mathbb{R}_{+} \backslash\{0,1,2, \ldots\}$ with the equivalent norm

$\|f\|_{\mathcal{C}^{\alpha}\left(\langle x\rangle^{\mu}\right)} \approx \sum_{|k| \leqslant\lfloor\alpha\rfloor} \sup _{x}\langle x\rangle^{\mu}\left|\partial^{k} f(x)\right|+\sum_{|k|=\lfloor\alpha\rfloor} \sup _{0<|x-y| \leqslant 1}\langle x\rangle^{\mu} \frac{\left|\partial^{k} f(x)-\partial^{k} f(y)\right|}{|x-y|^{\alpha-\lfloor\alpha\rfloor}}$.

We refer to [Tri06, sections 6.1 and 6.2] for these identities. A special case of (9) is the equivalence [Tri06, theorem 6.9]

$$
\|f\|_{H^{n}\left(\langle x\rangle^{\mu}\right)} \approx\|f\|_{L^{2}\left(\langle x\rangle^{\mu}\right)}+\sum_{0<|k| \leqslant n}\left\|\partial^{k} f\right\|_{L^{2}\left(\langle x\rangle^{\mu}\right)}
$$

for $n \in \mathbb{N}$. In particular we have for $n=0$ that $\mathcal{B}_{2,2}^{0}\left(\langle x\rangle^{\mu}\right)=H^{0}\left(\langle x\rangle^{\mu}\right)=L^{2}\left(\langle x\rangle^{\mu}\right)$. Restriction in (10) to $x, y \in \Omega, \Omega \subset \mathbb{R}^{d}$, and setting $\mu=0$ gives rise to the local Hölder space $\mathcal{C}^{\alpha}(\Omega)$. We can then bound (10) by

$$
\|f\|_{\mathcal{C}^{\alpha}\left(\langle x\rangle^{\mu}\right)} \leqslant C \sup _{k \in \mathbb{N}}\langle k\rangle^{\mu}\|f\|_{\mathcal{C}^{\alpha}\left([-k, k]^{2}\right)}
$$

for $\mu \in \mathbb{R}$ (with equivalence if $\mu \leqslant 0$ ).

We will use extensively the following interpolation result between weighted Besov spaces with mixed weights [SSV14, theorem 3.8].

Lemma 2.2. Let $p_{0}, q_{0}, p_{1}, q_{1} \in[1, \infty], \alpha_{0}, \alpha_{1}, \mu_{0}, \mu_{1} \in \mathbb{R}$. Let further $p, q, \alpha, \mu$ be such that there is a $\Theta \in[0,1]$ with $\frac{1}{p}=\frac{1-\Theta}{p_{0}}+\frac{\Theta}{p_{1}}, \frac{1}{q}=\frac{1-\Theta}{q_{0}}+\frac{\Theta}{q_{1}}, \alpha=(1-\Theta) \alpha_{0}+\Theta \alpha_{1}$ and $\mu=(1-\Theta) \mu_{0}+\Theta \mu_{1}$. It then holds

$$
\|f\|_{\mathcal{B}_{p, q}^{\alpha}\left(\langle x\rangle^{\mu}\right)} \leqslant C\|f\|_{\mathcal{B}_{p_{0}, q_{0}}^{\alpha_{0}}\left(\langle x\rangle^{\mu_{0}}\right)}^{1-\Theta}\|f\|_{\mathcal{B}_{p_{1}, q_{1}}^{\alpha_{1}}\left(\langle x\rangle^{\mu_{1}}\right)^{-}}^{\Theta} .
$$

Finally let us mention the following elementary inequality which we will use occasionally to close estimates: for any $x, y, \zeta>0$ and $\Theta \in(0,1)$ we have

$$
x^{1-\Theta} \cdot y^{\Theta} \leqslant \zeta \cdot x+\zeta^{-\frac{1-\Theta}{\Theta}} C_{\Theta} \cdot y
$$

with $C_{\Theta}:=(1-\Theta)^{\frac{1-\Theta}{\Theta}} \Theta$ (this is a scaled version of [Yos74, lemma I.3.1]).

\subsection{Growth of $\xi$ and $Y$}

We recall the following result on the growth of the white noise, which we take from [AC15, lemma 5.3].

Lemma 2.3. Let $\xi$ be white noise on $\mathbb{R}^{2}$ and pick uniformly bounded $\chi_{k} \in C^{2}\left(\mathbb{R}^{2}\right)$ with supp $\chi_{k} \subseteq[-k-1, k+1]^{2}$ and $\left.\chi_{k}\right|_{[-k, k]^{2}}=1$. For $\alpha<1$ there are $\lambda, \lambda^{\prime}>0$ such that

$$
\sup _{k \in \mathbb{N}} \frac{\mathbb{E}\left[\exp \left(\lambda\left\|\xi \chi_{k}\right\|_{\mathcal{C}^{\alpha-2}\left(\mathbb{R}^{2}\right)}^{2}\right)\right.}{k^{\lambda^{\prime}}}<\infty \text {. }
$$

Remark 2.4. In [AC15] the authors actually bound periodic white noise $\xi_{k}$ on $[-k, k]^{2}$ instead of $\chi_{k} \xi$. However this can be easily translated into the result above due to $\xi_{k+1} \chi_{k} \stackrel{\stackrel{d}{=}}{=} \xi \chi_{k}$ and $\left\|\chi_{k} \xi_{k+1}\right\|_{\mathcal{C}^{\alpha-2}\left(\mathbb{R}^{2}\right)} \leqslant C\left\|\xi_{k+1}\right\|_{\mathcal{C}^{\alpha-2}\left([-k-1, k+1]^{2}\right)}$ for $\alpha \in(0,1)$. 
We now proceed as in [HL15] and use a truncated Green's function $G \in C^{\infty}\left(\mathbb{R}^{2} \backslash\{0\}\right)$ that satisfies supp $G \subseteq B(0,1)$ (the unit ball around 0 ) and $G(x)=\frac{1}{2 \pi} \log |x|$ for $|x|$ small enough, so that $Y:=G * \xi$, with $\xi$ being white noise on $\mathbb{R}^{2}$ as in lemma 2.3 , solves

$$
\Delta Y=\xi+\varphi * \xi
$$

for some $\varphi \in C_{c}^{\infty}\left(\mathbb{R}^{2}\right)$ (the set of smooth, compactly supported functions).

As in [HL15] we introduce the random variable

$$
: \nabla Y^{2}::=\int_{\mathbb{R}^{2}} \int_{\mathbb{R}^{2}} \nabla G\left(\cdot-z_{1}\right) \nabla G\left(\cdot-z_{2}\right) \boldsymbol{\xi}\left(\mathrm{d} z_{1}\right) \boldsymbol{\xi}\left(\mathrm{d} z_{2}\right)
$$

with $\boldsymbol{\xi}$ denoting the Gaussian stochastic measure on $\mathbb{R}^{2}$ induced by the white noise $\xi$, compare [Jan97, p 95-99]. Relation (15) should be read in the distributional sense, i.e. for $\phi \in \mathcal{S}\left(\mathbb{R}^{2}\right)$ we have

$$
: \nabla Y^{2}:(\phi)=\int_{\mathbb{R}^{2}} \int_{\mathbb{R}^{2}}\left(\int_{\mathbb{R}^{2}} \phi(x) \nabla G\left(x-z_{1}\right) \nabla G\left(x-z_{2}\right) \mathrm{d} x\right) \boldsymbol{\xi}\left(\mathrm{d} z_{1}\right) \boldsymbol{\xi}\left(\mathrm{d} z_{2}\right)
$$

so that : $\nabla Y^{2}$ : is only defined (almost surely) as a distribution. Recall, that for $f_{1}, f_{2} \in L^{2}\left(\mathbb{R}^{2}\right)$ we have the following identity for $X_{1}:=\int_{\mathbb{R}^{2}} f_{1}\left(z_{1}\right) \boldsymbol{\xi}\left(\mathrm{d} z_{1}\right), X_{2}:=\int_{\mathbb{R}^{2}} f_{2}\left(z_{2}\right) \boldsymbol{\xi}\left(\mathrm{d} z_{2}\right)$ (compare [Jan97, theorem 7.26])

$$
: X_{1} \cdot X_{2}:=\int_{\mathbb{R}^{2}} \int_{\mathbb{R}^{2}} f_{1}\left(z_{1}\right) f_{2}\left(z_{2}\right) \boldsymbol{\xi}\left(\mathrm{d} z_{1}\right) \boldsymbol{\xi}\left(\mathrm{d} z_{2}\right)
$$

where $: X_{1} \cdot X_{2}$ : denotes the Wick product between the Gaussian random variables $X_{1}, X_{2}$, so that from this perspective definition $: \nabla Y^{2}$ : can be read as a 'Wick product' of the distribution $\nabla Y$ with itself. For an introduction to Wick calculus let us refer to [HKPS93, Jan97, Nua06]. The smoothness of $Y$ and $: \nabla Y^{2}$ : is described by the following lemma. Roughly speaking, all the results used in the case of the torus in [DW18] generalize except that due to the growth of the white noise we need to introduce weights in the norms we use.

Lemma 2.5 (Taken from [HL15]). For any $p \in[1, \infty), \delta>0$ and $\alpha \in(0,1)$ we have

$$
\mathbb{E}\left[\|\xi\|_{\mathcal{C}^{\alpha-2}\left(\langle x\rangle^{-\delta}\right)}^{p}+\|Y\|_{\mathcal{C}^{\alpha}\left(\langle x\rangle^{-\delta}\right)}^{p}+\left\|: \nabla Y^{2}:\right\|_{\mathcal{C}^{\alpha-1}(\langle x\rangle-\delta)}^{p}\right]<\infty .
$$

The weights used in lemma 2.5 are in fact too strong, because, as we can see from lemma 2.3, the actual growth of the noise and its derived quantities are really of the order $(\sqrt{\log |x|})^{1+\kappa}$ for $\kappa>0$. However, the polynomial weights will be completely sufficient for our purposes with the only exception in the following corollary of lemma 2.3, that exploits the sub-logarithmic growth of $Y$.

Corollary 2.6. For any $a \in \mathbb{R}, \alpha \in(0,1), \delta>0$ and $p \in[1, \infty)$ we have

$$
\mathbb{E}\left[\left\|\mathrm{e}^{a Y}\right\|_{\mathcal{C}^{\alpha}(\langle x\rangle-\delta)}^{p}\right]<\infty .
$$

Proof. Note first that, using (12), we can bound

$$
\left\|\mathrm{e}^{a Y}\right\|_{\mathcal{C}^{\alpha}\left(\langle x\rangle^{-\delta}\right)} \leqslant C \sup _{k \in \mathbb{N}} \frac{\left\|\mathrm{e}^{a Y}\right\|_{\mathcal{C}^{\alpha}\left([-k, k]^{2}\right)}}{k^{\delta}} \leqslant C \sup _{k \in \mathbb{N}} \frac{\exp \left(C|a|\|Y\|_{\mathcal{C}^{\alpha}\left([-k, k]^{2}\right)}\right)}{k^{\delta}},
$$


so that it remains to bound the $p$ th moment of the right hand side. Using the compact support of the Green's function we see

$$
\|Y\|_{\mathcal{C}^{\alpha}\left([-k, k]^{2}\right)} \leqslant C\left\|\chi_{k+2} \xi\right\|_{\mathcal{C}^{\alpha-2}\left(\mathbb{R}^{2}\right)}
$$

(to show this one can for example use the wavelet characterization of Besov spaces, as in [Tri06], and a decomposition of $G$ as in [Hai14, remark 5.6]). With lemma 2.3 we can therefore find $\lambda, \lambda^{\prime}>0$ such that

$$
\sup _{k \in \mathbb{N}} \frac{\mathbb{E}\left[\exp \left(\lambda\|Y\|_{\mathcal{C}^{\alpha}\left([-k, k]^{2}\right)}^{2}\right)\right]}{k^{\lambda^{\prime}}}<\infty .
$$

We now pick, without loss of generality, $p \in[1, \infty)$ so big that $p \delta \geqslant 2+\lambda^{\prime}$ which gives us

$$
\begin{aligned}
\mathbb{E}\left[\left|\sup _{k \in \mathbb{N}} \frac{\exp \left(C|a|\|Y\|_{\mathcal{C}^{\alpha}\left([-k, k]^{2}\right)}\right)}{k^{\delta}}\right|^{p}\right] & \leqslant \sum_{k=1}^{\infty} \frac{\mathbb{E}\left[\exp \left(p C|a|\|Y\|_{\mathcal{C}^{\alpha}\left([-k, k]^{2}\right)}\right)\right]}{k^{\delta p}} \\
& \leqslant C \sum_{k=1}^{\infty} \frac{\mathbb{E}\left[\exp \left(\lambda\|Y\|_{\mathcal{C}^{\alpha}\left([-k, k]^{2}\right)}^{2}\right)\right]}{k^{2} \cdot k^{\lambda^{\prime}}}<\infty
\end{aligned}
$$

where we made use of $\exp (p C|a| \cdot x) \leqslant C \exp \left(\lambda \cdot x^{2}\right)$ in the last step.

Due to [BCD11, lemma 2.2] the term $\varphi * \xi$ in (14) is smooth with

$$
\|\varphi * \xi\|_{\mathcal{C}^{\beta}\left(\langle x\rangle^{-\delta}\right)} \leqslant C \cdot\|\xi\|_{\mathcal{C}^{\alpha-2}\left(\langle x\rangle^{-\delta}\right)}
$$

for any $\beta \in \mathbb{R}, \alpha \in(0,1)$ and $\delta>0$ (lemma 2.2 of [BCD11] is in fact stated for unweighted spaces, but using the same trick as in the proof of property $(v)$ of lemma 2.1 weights can be included).

We will mostly work with a mollification of the noise: fix a smooth, compactly supported mollifier $\rho \in C_{c}^{\infty}(B(0,1))$ with

$$
\rho \geqslant 0, \quad \int_{\mathbb{R}^{2}} \rho(x) \mathrm{d} x=1
$$

and define for $\varepsilon \in(0,1]$ and $\rho_{\varepsilon}:=\varepsilon^{-2} \rho\left(\varepsilon^{-1} \cdot\right)$ the smoothened noise

$$
\xi_{\varepsilon}:=\rho_{\varepsilon} * \xi, \quad Y_{\varepsilon}:=\rho_{\varepsilon} * Y=\rho_{\varepsilon} * G * \xi=G * \xi_{\varepsilon} .
$$

We have (with the same $\varphi \in C_{c}^{\infty}\left(\mathbb{R}^{2}\right)$ as above)

$$
\Delta Y_{\varepsilon}=\xi_{\varepsilon}+\varphi * \xi_{\varepsilon} .
$$

According to (16) we can express the Wick product of the function $\nabla Y_{\varepsilon}$ with itself via

$$
: \nabla Y_{\varepsilon}^{2}:=: \nabla Y_{\varepsilon} \cdot \nabla Y_{\varepsilon}:=\int_{\mathbb{R}^{2}} \int_{\mathbb{R}^{2}} \nabla G_{\varepsilon}\left(\cdot-z_{1}\right) \nabla G_{\varepsilon}\left(\cdot-z_{2}\right) \boldsymbol{\xi}\left(\mathrm{d} z_{1}\right) \boldsymbol{\xi}\left(\mathrm{d} z_{2}\right)
$$

where $G^{\varepsilon}:=\rho_{\varepsilon} * G$. Note that $: \nabla Y_{\varepsilon}^{2}:$ is really a function in contrast to the genuine distribution $: \nabla Y^{2}:$

For the next result, recall that we denote by $K_{\varepsilon}$ a random constant that is bounded, uniformly in $\varepsilon$, in $L^{p}(\mathbb{P})$ for any $p \in[1, \infty)$.

Lemma 2.7. We have for any $\delta>0, \alpha \in(0,1), \beta \in \mathbb{R}, a \in \mathbb{R}$

$$
\left\|Y_{\varepsilon}\right\|_{\mathcal{C}^{\alpha}\left(\langle x\rangle^{-\delta}\right)}+\left\|: \nabla Y_{\varepsilon}^{2}:\right\|_{\mathcal{C}^{\alpha-1}\left(\langle x\rangle^{-\delta}\right)}+\left\|\mathrm{e}^{a Y_{\varepsilon}}\right\|_{\mathcal{C}^{\alpha}\left(\langle x\rangle^{-\delta}\right)}+\left\|\varphi * \xi_{\varepsilon}\right\|_{\mathcal{C}^{\beta}\left(\langle x\rangle^{-\delta}\right)} \leqslant K_{\varepsilon}
$$


Proof. Note that the claim is equivalent to

$\mathbb{E}\left[\left\|Y_{\varepsilon}\right\|_{\mathcal{C}^{\alpha}\left(\langle x\rangle^{-\delta}\right)}^{p}+\left\|: \nabla Y_{\varepsilon}^{2}:\right\|_{\mathcal{C}^{\alpha-1}\left(\langle x\rangle^{-\delta}\right)}^{p}+\left\|\mathrm{e}^{a Y_{\varepsilon}}\right\|_{\mathcal{C}^{\alpha}\left(\langle x\rangle^{-\delta}\right)}^{p}+\left\|\varphi * \xi_{\varepsilon}\right\|_{\mathcal{C}^{\beta}\left(\langle x\rangle^{-\delta}\right)}^{p}\right] \leqslant C$,

for $p \in[1, \infty)$, where $C>0$ might depend on $p$ and $\Delta$ but not on $\varepsilon$. Indeed, we can simply take $K_{\varepsilon}$ to be

$K_{\varepsilon}=\left\|Y_{\varepsilon}\right\|_{\mathcal{C}^{\alpha}(\langle x\rangle-\delta)}+\left\|: \nabla Y_{\varepsilon}^{2}:\right\|_{\mathcal{C}^{\alpha-1}(\langle x\rangle-\delta)}+\left\|\mathrm{e}^{a Y_{\varepsilon}}\right\|_{\mathcal{C}^{\alpha}(\langle x\rangle-\delta)}+\left\|\varphi * \xi_{\varepsilon}\right\|_{\mathcal{C}^{\beta}(\langle x\rangle-\delta)}$.

$K_{\varepsilon}$ is then in any $L^{p}(\mathbb{P})$ (with a norm that can be bounded uniformly in $\varepsilon$ ) for $p<\infty$, provided that (21) holds due to the basic estimate $(a+b)^{p} \leqslant C\left(a^{p}+b^{p}\right)$ for $a, b>0$ and $C=C(p)>0$. From property $(v)$ of lemma 2.1 we see that $\left\|Y_{\varepsilon}\right\|_{\mathcal{C}^{\alpha}\left(\langle x\rangle^{-\delta}\right)}=$ $\left\|\rho_{\varepsilon} * Y\right\|_{\mathcal{C}^{\alpha}(\langle x\rangle-\delta)}\|\leqslant C\| Y \|_{\mathcal{C}^{\alpha}\left(\langle x\rangle^{-\delta}\right)}$ so that $\mathbb{E}\left[\left\|Y_{\varepsilon^{*}}\right\|_{\mathcal{C}^{\alpha}(\langle x\rangle-\delta)}^{p}\right]$ in (21) is bounded by lemma 2.5. The bound on $\mathbb{E}\left[\left\|\mathrm{e}^{a Y_{\varepsilon}}\right\|_{\mathcal{C}^{\alpha}(\langle x\rangle-\delta)}^{p}\right]$ can then be deduced as in corollary 2.6. The Wick product $: \nabla Y_{\varepsilon}^{2}$ : is handled in the proof of proposition 1.3 in [HL15]. Finally, the bound on the term in (21) involving $\varphi * \xi_{\varepsilon}=\rho_{\varepsilon} * \varphi * \xi$ follows from property (v) of lemma 2.1, inequality (17) and lemma 2.5 .

Let us also recall the following statements, again from [HL15].

Lemma 2.8. For $\alpha \in(0,1), \delta>0$ and $\kappa \in(0,1-\alpha)$ we have

$$
\left\|Y_{\varepsilon}-Y\right\|_{\mathcal{C}^{\alpha}\left(\langle x\rangle^{-\delta}\right)}+\left\|\widetilde{: \nabla Y_{\varepsilon}^{2}:}-\widetilde{: \nabla Y^{2}:}\right\|_{\mathcal{C}^{\alpha-1}\left(\langle x\rangle^{-\delta}\right)} \leqslant K_{\varepsilon} \varepsilon^{\kappa} .
$$

Remark 2.9. In [HL15] the authors consider $: \nabla Y_{\varepsilon}^{2}:-: \nabla Y^{2}:$ instead of $\widetilde{\nabla Y_{\varepsilon}^{2}}:-\widetilde{\nabla Y^{2}}$ :, for the contribution of $\varphi * \xi_{\varepsilon}-\varphi * \xi$ we use (24) below.

Together with the bounds (20) and corollary 2.6 we then obtain

$$
\mathbb{E}\left[\left\|\mathrm{e}^{a Y}-\mathrm{e}^{a Y_{\varepsilon}}\right\|_{\mathcal{C}^{\alpha}(\langle x\rangle-\delta)}^{p}\right] \leqslant C \varepsilon^{p \kappa}
$$

for $a \in \mathbb{R}, \alpha \in(0,1)$ and $\kappa \in(0,1-\alpha)$. Further we have for $\beta \in \mathbb{R}, \alpha \in(0,1) \delta>0$, $p \in[1, \infty)$ and $\kappa \in(0,1-\alpha)$

$\left\|\varphi * \xi_{\varepsilon}-\varphi * \xi\right\|_{\mathcal{C}^{\beta}\left(\langle x\rangle^{-\delta}\right)}=\left\|\varphi *\left(\xi_{\varepsilon}-\xi\right)\right\|_{\mathcal{C}^{\beta}\left(\langle x\rangle^{-\delta}\right)} \leqslant C\left\|\xi-\xi_{\varepsilon}\right\|_{\mathcal{C}^{\alpha-2}\left(\langle x\rangle^{-\delta}\right)} \leqslant K_{\varepsilon} \varepsilon^{\kappa}$

where we used once more [BCD11, lemma 2.2] and in the last step $\left\|\xi-\xi_{\varepsilon}\right\|_{\mathcal{C}^{\alpha-2}(\langle x\rangle-\delta)} \leqslant K_{\varepsilon} \varepsilon^{\kappa}$ due to (the proof of) [HL15, lemma 1.1]. It will turn out convenient to have an estimate on the blow-up of the $L^{p}$ norm of $\nabla Y$, which is covered by the following lemma.

Lemma 2.10. Given $\delta \in(0,1), p \in(2 / \delta, \infty)$ we have

$$
\left\|\nabla Y_{\varepsilon}\right\|_{L^{p}\left(\langle x\rangle^{-\delta}\right)}^{2}+\left\|: \nabla Y_{\varepsilon}^{2}:\right\|_{L^{p}(\langle\langle\rangle-\delta)} \leqslant K_{\varepsilon}|\log (\varepsilon)| .
$$

Proof. As in the proof of lemma 2.7 we can reduce the claim to

$$
\mathbb{E}\left[\left\|\nabla Y_{\varepsilon}\right\|_{L^{p}\left(\langle x\rangle^{-\delta}\right)}^{2 q}+\left\|: \nabla Y_{\varepsilon}^{2}:\right\|_{L^{p}\left(\langle x\rangle^{-\delta}\right)}^{q}\right] \leqslant C|\log (\varepsilon)|^{q}
$$

for $q \in[1, \infty)$ and $C=C(p, q, \delta)>0$. We take without loss generality $q \in[p, \infty)$ (which implies in particular $2 q>p$ ) and obtain via Jensen's inequality 
$\mathbb{E}\left[\left\|\nabla Y_{\varepsilon}\right\|_{L^{p}(\langle x\rangle-\delta)}^{2 q}+\left\|: \nabla Y_{\varepsilon}^{2}:\right\|_{L^{p}\left(\langle x\rangle^{-\delta}\right)}^{q}\right] \leqslant C \int_{\mathbb{R}^{2}} \frac{1}{\langle x\rangle p^{p}} \mathbb{E}\left[\left|\nabla Y_{\varepsilon}(x)\right|^{2 q}+\left|: \nabla Y_{\varepsilon}^{2}:(x)\right|^{q}\right] \mathrm{d} x$.

The result follows now from equivalence of moments [Jan97, theorem 3.50] and $\mathbb{E}\left[\left|\nabla Y_{\varepsilon}(x)\right|^{2}\right.$, $\mathbb{E}\left[: \nabla Y_{\varepsilon}^{2}:(x)\right] \leqslant C|\log \varepsilon|($ see $[$ HL15]).

\subsection{Set-up and conserved quantities}

We consider the equation

$$
\imath \partial_{t} u=\Delta u+u \xi+\lambda|u|^{2 \sigma} u, u(0)=u_{0},
$$

or rather a suitable transformed and renormalized version, which we introduce below.

It is well-known (see for example [Caz03]) that a solution to (25), if existent, has at least formally the conserved, that is time-independent, quantities mass $N(u(t))=N\left(u_{0}\right)$ and energy $H(u(t))=H\left(u_{0}\right)$ defined as

$$
\begin{aligned}
& N(u(t))=\int_{\mathbb{R}^{2}}|u(t, x)|^{2} \mathrm{~d} x, \\
& H(u(t))=\int_{\mathbb{R}^{2}}\left(\frac{1}{2}|\nabla u(t, x)|^{2}-\frac{1}{2}|u(t, x)|^{2} \xi-\frac{\lambda}{2 \sigma+2}|u(t, x)|^{2 \sigma+2}\right) \mathrm{d} x .
\end{aligned}
$$

Most of the analysis in this article ought to be read pathwise: although the white noise $\xi$ should be thought of as a random distribution, we solve (25) (or its transformed version below) for every event in the considered probability space, so that most of the techniques we apply are completely deterministic. Stochastic concepts are used in order to formulate the limits in theorems 4.4 and 5.2 below and, more importantly, in order to introduce a renormalization into (25). This way of proceeding, to only use probabilistic methods at the most delicate points in the treatment of a stochastic partial differential equation, has been applied frequently and quite successfully in the recent years, even in the treatment of 'singular' stochastic partial differential equations [CC18, DPD03, GIP15, Hai14, MP17, WM15] (by no means exhaustive). A typical issue that arises in such equations is the presence of an ill-defined term, typically the product of a distribution with a non-smooth function, which is in general not well-defined. In fact, we encounter the same complication in (25), as we do not know if the product between the (probably non-smooth) solution $u$ and the distribution $\xi$ is well-defined. Even though there are certain smoothness conditions under which such a product can still be constructed rigorously, this just fails to be the case for (25), compare [DW18].

We follow [DW18] in the idea to substitute $u$ in this equation by $v=\mathrm{e}^{Y} u$ with $Y$ introduced above and obtain the problem

$\imath \partial_{t} v=\Delta v+v\left(\nabla Y^{2}-\varphi * \xi\right)-2 \nabla v \nabla Y+\lambda|v|^{2 \sigma} v \mathrm{e}^{-2 \sigma Y}, v(0)=v_{0}:=\mathrm{e}^{Y} u_{0}$

with the conserved quantities

$$
\begin{aligned}
\tilde{N}(v(t)) & =N\left(\mathrm{e}^{-Y} v(t)\right)=\int_{\mathbb{R}^{2}}|v(t, x)|^{2} \mathrm{e}^{-2 Y(x)} \mathrm{d} x, \\
\tilde{H}(v(t)) & =H\left(\mathrm{e}^{-Y} v(t)\right) \\
& =\int_{\mathbb{R}^{2}}\left(\frac{1}{2}|\nabla v(t, x)|^{2}-\frac{1}{2}|v(t, x)|^{2} \widetilde{\nabla Y^{2}(x)}-\frac{\lambda}{2 \sigma+2}|v(t, x)|^{2 \sigma+2}\right) \mathrm{e}^{-2 Y(x)} \mathrm{d} x
\end{aligned}
$$

with $\widetilde{\nabla Y^{2}}=\nabla Y^{2}-\varphi * \xi$. 
The transformation $v=e^{Y} u$ did not really resolve our problem as we now have for instance a square $\nabla Y^{2}$ in (26) that is ill-defined: from lemma 2.5 we only get $\nabla Y \in \mathcal{C}_{\langle x\rangle-\kappa}^{0^{-}}$for $\kappa>0$ so that we expect $\nabla Y$ to be a distribution and thus $\nabla Y^{2}$ to be meaningless. A rather popular approach to obtain a well-defined equation is to perform a Wick renormalization [DPD03, HL15, DW18]. Namely, we consider instead of (26) the problem

$\imath \partial_{t} v=\Delta v+v\left(: \nabla Y^{2}:-\varphi * \xi\right)-2 \nabla v \nabla Y+\lambda|v|^{2 \sigma} v \mathrm{e}^{-2 \sigma Y}, v(0)=v_{0}:=\mathrm{e}^{Y} u_{0}$,

where the distribution $: \nabla Y^{2}$ : is defined above. Although this equation still exhibits the products $\nabla v \nabla Y$ and $v: \nabla Y^{2}$ : which can only be defined if $v$ is smooth enough, the 'noise' $: \nabla Y^{2}:$ and $\nabla Y$ is now contained a.s. in $\mathcal{C}^{0^{-}}\left(\langle x\rangle^{-\kappa}\right)$ and is thus much more regular than $\xi \in \mathcal{C}^{-1^{-}}\left(\langle x\rangle^{-\kappa}\right)$ and allows, as we will see, for a solution. The effect of the replacement $\nabla Y^{2} \rightarrow: \nabla Y^{2}$ : is more lucid in a framework where we avoid ill-defined expressions. Consider the mollification $Y_{\varepsilon}=\rho_{\varepsilon} * Y$ of $Y$ from (18) and the corresponding smoothened version of (27)

$$
\imath \partial_{t} v_{\varepsilon}=\Delta v_{\varepsilon}+v_{\varepsilon}: \widetilde{\nabla Y_{\varepsilon}^{2}}:-2 \nabla v_{\varepsilon} \nabla Y_{\varepsilon}+\lambda\left|v_{\varepsilon} \mathrm{e}^{-Y_{\varepsilon}}\right|^{2 \sigma} v_{\varepsilon}, v_{\varepsilon}(0)=v_{0}
$$

with $\widetilde{\nabla Y_{\varepsilon}^{2}}:=: \nabla Y_{\varepsilon}^{2}:-\varphi * \xi_{\varepsilon}$ and where we start (for simplicity) in the same initial condition $v_{0}$ as in (27). Recall that the object $: \nabla Y_{\varepsilon}^{2}$ :, given by (19), can now really be read as the Wick product of $\nabla Y_{\varepsilon}$ with itself, which is of the form $: \nabla Y_{\varepsilon}^{2}:=\left|\nabla Y_{\varepsilon}\right|^{2}-c_{\varepsilon}$ (compare [Jan97]), where $c_{\varepsilon}=\mathbb{E}\left[\left|\nabla Y_{\varepsilon}(x)\right|^{2}\right]=\int\left|\nabla G_{\varepsilon}(x-z)\right|^{2} \mathrm{~d} z=\int\left|\nabla G_{\varepsilon}(z)\right|^{2} \mathrm{~d} z \sim|\log \varepsilon|$ (as in [HL15, p 3]). Considering now $u_{\varepsilon}:=v_{\varepsilon} \mathrm{e}^{-Y_{\varepsilon}}$ we obtain the mollified and renormalized version of (25)

$$
\imath \partial_{t} u_{\varepsilon}=\Delta u_{\varepsilon}+u_{\varepsilon}\left(\xi_{\varepsilon}-c_{\varepsilon}\right)+\lambda\left|u_{\varepsilon}\right|^{2 \sigma} u_{\varepsilon}, u_{\varepsilon}(0)=\mathrm{e}^{-Y_{\varepsilon}} v_{0},
$$

so that the effect of the Wick renormalization above consists in the cancellation of a diverging phase $\mathrm{e}^{2 c_{\varepsilon} t}$ from the solution of (the mollified version of) (25).

The aim of this article is to construct a solution $v$ to (27) as the limit of the mollified equation

$$
\imath \partial_{t} v_{\varepsilon}=\Delta v_{\varepsilon}+v_{\varepsilon}: \widetilde{\nabla Y_{\varepsilon}^{2}}:-2 \nabla v_{\varepsilon} \nabla Y_{\varepsilon}+\lambda\left|v_{\varepsilon} \mathrm{e}^{-Y_{\varepsilon}}\right|^{2 \sigma} v_{\varepsilon}, v_{\varepsilon}(0)=v_{0} .
$$

We assume from now on that the initial condition $v_{0}$ is independent of $\varepsilon$ and satisfies $v_{0} \in H^{2}\left(\langle x\rangle^{\delta_{0}}\right)$ a.s. for some fixed $\delta_{0} \in\left(0, \frac{1}{2}\right)$. Note that we allow $v_{0}$ to be random.

At this stage, we do not know whether equation (29) has a unique solution. This is due to the fact that the equation contains unbounded terms.

The next result states existence and uniqueness for the mollified equation. The presence of the unbounded potential $\xi_{\varepsilon}$ in (28) prevents the use of classical results. The proof is sketched in the appendix.

Proposition 2.11. Assume that $v_{0} \in H^{2}\left(\langle x\rangle^{\delta_{0}}\right)$ a.s. When $\lambda \leqslant 0$, there exists a random time $\tau_{0}$ such that, for any $\varepsilon>0$, equation (29) has a unique solution in the space $L^{\infty}\left(0, \tau_{0} ; H^{2}\left(\langle x\rangle^{-\delta}\right) \cap C_{\tau_{0}} H^{\gamma}\left(\langle x\rangle^{\delta^{\prime}}\right)\right.$ for any $\delta>0, \gamma \in(1,2)$ and $\delta^{\prime}<\left(1-\frac{\gamma}{2}\right) \delta_{0}$.

When $\sigma<1$ and $\lambda \in \mathbb{R}$, for any $\varepsilon>0$ and $T \geqslant 0$, equation (29) has a unique solution in the space $L^{\infty}\left(0, T ; H^{2}\left(\langle x\rangle^{-\delta}\right) \cap C_{T} H^{\gamma}\left(\langle x\rangle^{\delta^{\prime}}\right)\right.$ for any $\delta>0, \gamma \in(1,2)$ and $\delta^{\prime}<\left(1-\frac{\gamma}{2}\right) \delta_{0}$.

Also, the proof shows that the following quantities (which we again call mass and energy) are conserved:

$$
\begin{aligned}
& \tilde{N}_{\varepsilon}\left(v_{\varepsilon}(t)\right):=\int_{\mathbb{R}^{2}}\left|v_{\varepsilon}(t, x)\right|^{2} \mathrm{e}^{-2 Y_{\varepsilon}(x)} \mathrm{d} x \\
& \tilde{H}_{\varepsilon}\left(v_{\varepsilon}(t)\right):=\int_{\mathbb{R}^{2}}\left(\frac{1}{2}\left|\nabla v_{\varepsilon}(t, x)\right|^{2}-\frac{1}{2}\left|v_{\varepsilon}(t, x)\right|^{2}: \widetilde{\nabla Y_{\varepsilon}^{2}(x)}:-\frac{\lambda}{2 \sigma+2}\left|v_{\varepsilon}(t, x)\right|^{2 \sigma+2} \mathrm{e}^{-2 \sigma Y_{\varepsilon}(x)}\right) \mathrm{e}^{-2 Y_{\varepsilon}(x)} \mathrm{d} x .
\end{aligned}
$$


These expressions are well-defined due to lemma 2.7 and $v$ has values in $H^{\gamma}\left(\langle x\rangle^{\delta^{\prime}}\right)$ for any $\gamma \in(1,2)$ and $\delta^{\prime}<\left(1-\frac{\gamma}{2}\right) \delta_{0}$. Indeed for $t \in[0, T]$ we then have by (11) that $v_{\varepsilon}(t), \nabla v_{\varepsilon}(t) \in L^{2}\left(\langle x\rangle^{\delta^{\prime}}\right)$ and by the Sobolev embedding in lemma 2.1 further $v_{\varepsilon}(t) \in L^{2 \sigma+2}\left(\langle x\rangle^{\delta^{\prime}}\right)$. Since by lemma 2.7 and (10) it holds $\mathrm{e}^{a Y_{\varepsilon}}, \varphi * \xi_{\varepsilon} \in L^{\infty}\left(\langle x\rangle^{-\kappa}\right)$ for any $a \in \mathbb{R}$ and $\kappa>0$ this shows the well-defined character of all terms appearing above except for

$$
\int_{\mathbb{R}^{2}} \frac{1}{2}\left|v_{\varepsilon}(t, x)\right|^{2}: \nabla Y_{\varepsilon}^{2}(x): \mathrm{e}^{-2 Y_{\varepsilon}(x)} \mathrm{d} x,
$$

which we can handle by duality ( (iii) of lemmas 2.1) and 2.7:

$$
\begin{aligned}
\left.\left|\int_{\mathbb{R}^{2}} \frac{1}{2}: \nabla Y_{\varepsilon}^{2}(x): \cdot\right| v_{\varepsilon}(t, x)\right|^{2} \mathrm{e}^{-2 Y_{\varepsilon}(x)} \mathrm{d} x \mid & \leqslant C\left\|: \nabla Y_{\varepsilon}^{2}:\right\|_{\mathcal{C}^{-\beta}\left(\langle x\rangle^{-\delta^{\prime}}\right)} \cdot\left\|\left|v_{\varepsilon}(t)\right|^{2} \mathrm{e}^{-2 Y_{\varepsilon}}\right\|_{\mathcal{B}_{1,1}^{\beta}\left(\langle x\rangle^{\delta^{\prime}}\right)} \\
& \leqslant K_{\varepsilon}\left\|\left|v_{\varepsilon}(t)\right|^{2} \mathrm{e}^{-2 Y_{\varepsilon}}\right\|_{\mathcal{B}_{1,1}^{\beta}\left(\langle x\rangle^{\delta^{\prime}}\right)}
\end{aligned}
$$

with some arbitrary $\beta \in(0,1)$. The right hand side is finite by the multiplication bound (iv) in lemma 2.1:

$$
\begin{aligned}
\left\|\left|v_{\varepsilon}(t)\right|^{2} \mathrm{e}^{-2 Y_{\varepsilon}}\right\|_{\mathcal{B}_{1,1}^{\beta}\left(\langle x\rangle^{\delta^{\prime}}\right)} & \leqslant C\left\|\left|v_{\varepsilon}(t)\right|^{2}\right\|_{\mathcal{B}_{1,1}^{\beta+\kappa}\left(\langle x\rangle^{2 \delta^{\prime}}\right)} \cdot\left\|\mathrm{e}^{-2 Y_{\varepsilon}}\right\|_{\mathcal{C}^{\beta+\kappa}\left(\langle x\rangle^{-\delta^{\prime}}\right)} \\
& \leqslant C\left\|v_{\varepsilon}(t)\right\|_{H^{\beta+\kappa}\left(\langle x\rangle^{\delta^{\prime}}\right)}^{2} \cdot\left\|\mathrm{e}^{-2 Y_{\varepsilon}}\right\|_{\mathcal{C}^{\beta+\kappa}\left(\langle x\rangle-\delta^{\prime}\right)}
\end{aligned}
$$

for some arbitrary $\kappa \in(0,1-\beta)$ (which we need for the application of (iv) in lemma 2.1). Since $\beta+\kappa<1$ we have in particular $\left\|v_{\varepsilon}(t)\right\|_{H^{\beta+\kappa}\left(\langle x\rangle^{\delta^{\prime}}\right)} \leqslant C\left\|v_{\varepsilon}\right\|_{C_{T} H^{\gamma}\left(\langle x\rangle^{\delta}\right)}$ for $\gamma \in(1,2)$ and the well-defined character of the quantities above follows.

\section{Moments and a priori bound in $H^{1}$}

We start by a lemma that allows us to control moments of $v_{\varepsilon}$ by its derivatives. In this section we work in the framework and under the assumptions of proposition 2.11 , the estimates are stated on interval of time $[0, T]$. When $\sigma \geqslant 1$ and $\lambda \leqslant 0$, we assume implicitely that $T \leqslant \tau_{0}$. If $\sigma<1, T \geqslant 0$ is arbitrary.

Lemma 3.1. Let $v_{\varepsilon}$ be the unique solution to (29) on $[0, T]$ for some $T>0$. We then have for any $\delta \in\left(0, \delta_{0}\right)$ and $\delta^{\prime}<1-2 \delta$

$$
\left\|v_{\varepsilon}\right\|_{C_{T} L^{2}\left(\langle x\rangle^{\delta}\right)} \leqslant K_{\varepsilon}\left(1+\left\|v_{0}\right\|_{L^{2}\left(\langle x\rangle^{\delta_{0}}\right)}\right)\left(1+\left\|v_{\varepsilon}\right\|_{C_{T} H^{1}\left(\langle x\rangle^{-\delta^{\prime}}\right)}^{\frac{1}{2}}\right) .
$$

Proof. Observe that for $t \in[0, T]$

$$
\begin{aligned}
& \frac{\mathrm{d}}{\mathrm{d} t}\left(\int_{\mathbb{R}^{2}}\left|\langle x\rangle^{\delta} v_{\varepsilon}(\cdot, x)\right|^{2} \mathrm{e}^{-2 Y_{\varepsilon}(x)} \mathrm{d} x\right)(t)=2 \operatorname{Re}\left(\int_{\mathbb{R}^{2}}\langle x\rangle^{2 \delta} \partial_{t} v_{\varepsilon}(t, x) \cdot \bar{v}_{\varepsilon}(t, x) \mathrm{e}^{-2 Y_{\varepsilon}(x)} \mathrm{d} x\right) \\
& =2 \operatorname{Im}\left(\int_{\mathbb{R}^{2}}\langle x\rangle^{2 \delta}\left(\Delta v_{\varepsilon}(t, x)-2 \nabla v_{\varepsilon}(t, x) \cdot \nabla Y_{\varepsilon}(x)\right) \bar{v}_{\varepsilon}(t, x) \mathrm{e}^{-2 Y_{\varepsilon}(x)} \mathrm{d} x\right) \\
& =-2 \operatorname{Im}\left(\int_{\mathbb{R}^{2}} \nabla\langle x\rangle^{2 \delta} \cdot \nabla v_{\varepsilon}(t, x) \bar{v}_{\varepsilon}(t, x) \mathrm{e}^{-2 Y_{\varepsilon}(x)} \mathrm{d} x\right) \\
& \leqslant C \int_{\mathbb{R}^{2}}\langle x\rangle^{2 \delta-1}\left|\nabla v_{\varepsilon}(t, x)\right|\left|v_{\varepsilon}(t, x)\right| \mathrm{e}^{-2 Y_{\varepsilon}(x)} \mathrm{d} x
\end{aligned}
$$


Integrating for $t \in[0, T]$ over $[0, t]$ then shows with $\delta_{1}:=\delta_{0}-\delta$ and $\delta_{2}:=(1-2 \delta)-\delta^{\prime}$

$$
\begin{aligned}
& \sup _{t \in[0, T]} \int_{\mathbb{R}^{2}}\left|\langle x\rangle^{\delta} v_{\varepsilon}(t, x)\right|^{2} \mathrm{e}^{-2 Y_{\varepsilon}(x)} \mathrm{d} x \\
& \leqslant \int_{\mathbb{R}^{2}}\left|\langle x\rangle^{\delta} v_{0}(x)\right|^{2} \mathrm{e}^{-2 Y_{\varepsilon}(x)}+C \sup _{t \in[0, T]} \int_{\mathbb{R}^{2}}\langle x\rangle^{2 \delta-1}\left|\nabla v_{\varepsilon}(t, x)\right|\left|v_{\varepsilon}(t, x)\right| \mathrm{e}^{-2 Y_{\varepsilon}(x)} \mathrm{d} x \\
& \leqslant\left\|v_{0}\right\|_{L^{2}\left(\langle x\rangle^{\delta_{0}}\right)}^{2}\left\|\mathrm{e}^{-2 Y_{\varepsilon}}\right\|_{L^{\infty}\left(\langle x\rangle^{-\delta_{1}}\right)} \\
& +C \sup _{t \in[0, T]} \int_{\mathbb{R}^{2}}\langle x\rangle^{-\delta^{\prime}}\left|\nabla v_{\varepsilon}(t, x)\right|\left|v_{\varepsilon}(t, x)\right| \mathrm{e}^{-Y_{\varepsilon}(x)} \mathrm{d} x\left\|\mathrm{e}^{-Y_{\varepsilon}}\right\|_{L^{\infty}\left(\langle x\rangle^{-\delta_{2}}\right)} .
\end{aligned}
$$

With (10) and lemma 2.7 for some arbitrary $\alpha \in(0,1)$ we can bound the exponential factors: $\left\|\mathrm{e}^{-2 Y_{\varepsilon}}\right\|_{L^{\infty}\left(\langle x\rangle^{-\delta_{1}}\right)}+\left\|\mathrm{e}^{-Y_{\varepsilon}}\right\|_{L^{\infty}\left(\langle x\rangle^{-\delta_{2}}\right)} \leqslant C\left(\left\|\mathrm{e}^{-2 Y_{\varepsilon}}\right\|_{\mathcal{C}^{\alpha}\left(\langle x\rangle^{-\delta_{1}}\right)}+\left\|\mathrm{e}^{-Y_{\varepsilon}}\right\|_{\mathcal{C}^{\alpha}\left(\langle x\rangle^{\left.-\delta_{2}\right)}\right.}\right) \leqslant K_{\varepsilon}$.

By an application of the Cauchy-Schwarz inequality and conservation of mass we then obtain $\sup _{t \in[0, T]} \int_{\mathbb{R}^{2}}\left|\langle x\rangle^{\delta} v_{\varepsilon}(t, x)\right|^{2} \mathrm{~d} x \leqslant K_{\varepsilon}\left\|v_{0}\right\|_{L^{2}\left(\langle x\rangle^{\left.\delta_{0}\right)}\right.}^{2}+K_{\varepsilon} \sqrt{\tilde{N}_{\varepsilon}\left(v_{0}\right)}\|\nabla v\|_{C_{T} L^{2}\left(\langle x\rangle-\delta^{\prime}\right)}$

Applying once more lemma 2.7 to estimate $\left\|\mathrm{e}^{-Y_{\varepsilon}}\right\|_{L^{\infty}\left(\langle x\rangle-\delta_{0}\right)} \leqslant K_{\varepsilon}$ we obtain $\sqrt{\tilde{N}_{\varepsilon}\left(v_{0}\right)}=\left(\int_{\mathbb{R}^{2}}\left|v_{0}(x)\right|^{2} \mathrm{e}^{-2 Y_{\varepsilon}(x)} \mathrm{d} x\right)^{1 / 2} \leqslant K_{\varepsilon}\left\|v_{0}\right\|_{L^{2}\left(\langle x\rangle^{\delta_{0}}\right)} \leqslant K_{\varepsilon}\left(1+\left\|v_{0}\right\|_{L^{2}\left(\langle x\rangle^{\delta_{0}}\right)}\right)^{2}$.

Inserting (31) into the right hand side of (30), applying (11) and taking finally the square root closes the proof.

We now derive an $H^{1}$ bound for $v_{\varepsilon}$. This is essentially based on an energy estimate, similar as done in [DW18], but using lemma 3.1 to control appearing moments.

Proposition 3.2. Let $v_{\varepsilon}$ be the unique solution of (29) with $\lambda \leqslant 0$ or $\sigma<1$ on $[0, T]$ for $T>0$. We then have for any $\delta>0$

$$
\left\|v_{\varepsilon}\right\|_{C_{T} H^{1}\left(\langle x\rangle^{-\delta}\right)} \leqslant K_{\varepsilon}\left(1+\left\|v_{0}\right\|_{H^{1}\left(\langle x\rangle^{\left.\delta_{0}\right)}\right.}^{a}\right)
$$

for some deterministic $a>0$.

Proof. Note that it is sufficient to prove the claim for small $\delta>0$. According to (11) we have to bound $\left\|v_{\varepsilon}\right\|_{L^{2}(\langle x\rangle-\delta)}$ and $\left\|\nabla v_{\varepsilon}\right\|_{L^{2}(\langle x\rangle-\delta)}$. Note first that by conservation of mass for $t \in[0, T]$

$\left\|v_{\varepsilon}(t)\right\|_{L^{2}\left(\langle x\rangle^{-\delta}\right)}^{2}=\int_{\mathbb{R}^{2}}\left|v_{\varepsilon}(t, x)\right|^{2}\langle x\rangle^{-2 \delta} \mathrm{d} x \leqslant\left\|\mathrm{e}^{2 Y_{\varepsilon}}\right\|_{L^{\infty}\left(\langle x\rangle^{-2 \delta}\right)} \int_{\mathbb{R}^{2}}\left|v_{\varepsilon}(t, x)\right|^{2} \mathrm{e}^{-2 Y_{\varepsilon}(x)} \mathrm{d} x$

$=\left\|\mathrm{e}^{2 Y_{\varepsilon}}\right\|_{L^{\infty}\left(\langle x\rangle^{-2 \delta}\right)} \int_{\mathbb{R}^{2}}\left|v_{0}(x)\right|^{2} \mathrm{e}^{-2 Y_{\varepsilon}(x)} \mathrm{d} x$

$\leqslant\left\|\mathrm{e}^{2 Y_{\varepsilon}}\right\|_{L^{\infty}(\langle x\rangle-2 \delta)}\left\|\mathrm{e}^{-2 Y_{\varepsilon}}\right\|_{L^{\infty}\left(\langle x\rangle^{-2 \delta_{0}}\right)} \int_{\mathbb{R}^{2}}\left|v_{0}(x)\right|^{2}\langle x\rangle^{2 \delta_{0}} \mathrm{~d} x$.

Using lemma 2.7 and (10) we can bound the factor $\left\|\mathrm{e}^{2 Y_{\varepsilon}}\right\|_{L^{\infty}(\langle x\rangle-2 \delta)}\left\|\mathrm{e}^{-2 Y_{\varepsilon}}\right\|_{L^{\infty}\left(\langle x\rangle^{\left.-2 \delta_{0}\right)}\right.}$ and obtain the desired $L^{2}$ bound: 


$$
\left\|v_{\varepsilon}\right\|_{C_{T} L^{2}(\langle x\rangle-\delta)} \leqslant K_{\varepsilon}\left\|v_{0}\right\|_{L^{2}\left(\langle x\rangle \delta_{0}\right)} .
$$

It remains to find a bound on $\left\|\nabla v_{\varepsilon}\right\|_{L^{2}(\langle x\rangle-\delta)}$. By conservation of energy we obtain for $t \in[0, T]$

$$
\begin{aligned}
\int_{\mathbb{R}^{2}}\left|\nabla v_{\varepsilon}(t, x)\right|^{2} \mathrm{e}^{-2 Y_{\varepsilon}(x)} \mathrm{d} x= & 2 \tilde{H}_{\varepsilon}\left(v_{0}\right) \\
& +\int_{\mathbb{R}^{2}}\left(\left|v_{\varepsilon}(t, x)\right|^{2}: \widetilde{\nabla Y_{\varepsilon}^{2}(x)}:+c_{\lambda}\left|v_{\varepsilon}(t, x)\right|^{2 \sigma+2} \mathrm{e}^{-2 \sigma Y_{\varepsilon}(x)}\right) \mathrm{e}^{-2 Y_{\varepsilon}(x)} \mathrm{d} x,
\end{aligned}
$$

with $c_{\lambda}=\lambda /(\sigma+1)$. We estimate the first part of the integral on the right hand side by duality, Besov multiplication rules (property (iii) and (iv) in lemma 2.1) and lemma 2.7,

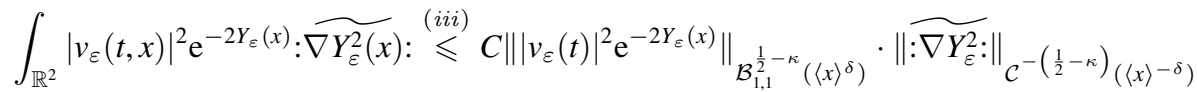

$$
\begin{aligned}
& \stackrel{(i v)}{\leqslant} K_{\varepsilon}\left\|v_{\varepsilon}(t)\right\|_{H^{\frac{1}{2}}\left(\langle x\rangle^{\delta / 2}\right)}^{2}
\end{aligned}
$$

where $\kappa \in\left(0, \frac{1}{2}\right)$. We can choose, without loss of generality, $\Delta$ small enough such that $2 \delta<\delta_{0}$ and $\delta<1-2 \delta$. We can then apply the interpolation result from lemmas 2.2 (with $\Theta=1 / 2$ ) and $3.1\left(\right.$ with $\delta^{\prime}=\delta$ )

$$
\begin{aligned}
&\left\|v_{\varepsilon}(t)\right\|_{H^{\frac{1}{2}}\left(\langle x\rangle^{\delta / 2}\right)}^{2} \stackrel{\text { lemma } 2.2}{\leqslant} C\left\|v_{\varepsilon}(t)\right\|_{L^{2}\left(\langle x\rangle^{2 \delta}\right)}\left\|v_{\varepsilon}(t)\right\|_{H^{1}(\langle x\rangle-\delta)} \\
& \stackrel{\text { lemma 3.1 }}{\leqslant} K_{\varepsilon}\left(1+\left\|v_{0}\right\|_{L^{2}\left(\langle x\rangle^{\delta}\right)}\right)\left(1+\left\|v_{\varepsilon}\right\|_{C_{T} H^{1}(\langle x\rangle-\delta)}^{3 / 2}\right) .
\end{aligned}
$$

Inserting into (33) we end up with

$$
\begin{aligned}
\int_{\mathbb{R}^{2}}\left|\nabla v_{\varepsilon}(t, x)\right|^{2} \mathrm{e}^{-2 Y_{\varepsilon}(x)} \mathrm{d} x & \leqslant 2 \tilde{H}_{\varepsilon}\left(v_{0}\right)+c_{\lambda} \int_{\mathbb{R}^{2}}\left|v_{\varepsilon}(t, x)\right|^{2 \sigma+2} \mathrm{e}^{-(2 \sigma+2) Y_{\varepsilon}(x)} \mathrm{d} x \\
& +K_{\varepsilon}\left(1+\left\|v_{0}\right\|_{L^{2}\left(\langle x\rangle^{\delta_{0}}\right)}\right)\left(1+\left\|v_{\varepsilon}\right\|_{C_{T} H^{1}(\langle x\rangle-\delta)}^{3 / 2}\right)
\end{aligned}
$$

Since $\tilde{H}_{\varepsilon}\left(v_{0}\right) \leqslant K_{\varepsilon}\left(1+\left\|v_{0}\right\|_{H^{1}\left(\langle x\rangle^{\delta}\right)}^{a}\right)$ for some $a>0$ (by the arguments at the end of section 2.3) we can write estimate into a more concise form

$$
\begin{aligned}
\int_{\mathbb{R}^{2}}\left|\nabla v_{\varepsilon}(t, x)\right|^{2} \mathrm{e}^{-2 Y_{\varepsilon}(x)} \mathrm{d} x & \leqslant c_{\lambda} \int_{\mathbb{R}^{2}}\left|v_{\varepsilon}(t, x)\right|^{2 \sigma+2} \mathrm{e}^{-(2 \sigma+2) Y_{\varepsilon}(x)} \mathrm{d} x \\
& +K_{\varepsilon}\left(1+\left\|v_{0}\right\|_{L^{2}\left(\left\langle x \delta^{\delta_{0}}\right)\right.}^{a}\right)\left(1+\left\|v_{\varepsilon}\right\|_{C_{T} H^{1}(\langle x\rangle-\delta)}^{3 / 2}\right)
\end{aligned}
$$

where we allow $a>0$ to change from line to line. We can bound the left hand side of this inequality from below by noting that due to lemma $2.7\left\|\mathrm{e}^{2 Y_{\varepsilon}}\right\|_{L^{\infty}\left(\langle x\rangle^{\left.-2 \delta_{0}\right)}\right.} \leqslant K_{\varepsilon}$ so that $\left\|\nabla v_{\varepsilon}(t)\right\|_{L^{2}(\langle x\rangle-\delta)}^{2} \leqslant K_{\varepsilon} \int_{\mathbb{R}^{2}}\left|\nabla v_{\varepsilon}(t, x)\right|^{2} \mathrm{e}^{-2 Y_{\varepsilon}(x)} \mathrm{d} x$ and we obtain

$$
\begin{aligned}
\left\|\nabla v_{\varepsilon}(t)\right\|_{L^{2}(\langle x\rangle-\delta)}^{2} & \leqslant c_{\lambda} K_{\varepsilon} \int_{\mathbb{R}^{2}}\left|v_{\varepsilon}(t, x)\right|^{2 \sigma+2} \mathrm{e}^{-(2 \sigma+2) Y_{\varepsilon}(x)} \mathrm{d} x \\
& +K_{\varepsilon}\left(1+\left\|v_{0}\right\|_{L^{2}\left(\langle x\rangle^{\delta}\right)}^{a}\right)\left(1+\left\|v_{\varepsilon}\right\|_{C_{T} H^{1}(\langle x\rangle-\delta)}^{3 / 2}\right) .
\end{aligned}
$$


Together with (11) and (32), after taking the supremum over $t \in[0, T]$

$$
\begin{aligned}
& \left\|v_{\varepsilon}\right\|_{C_{T} H^{1}\left(\langle x\rangle^{-\delta}\right)}^{2} \stackrel{(11) \varepsilon(32)}{\leqslant} c_{\lambda} K_{\varepsilon} \sup _{t \in[0, T]} \int_{\mathbb{R}^{2}}\left|v_{\varepsilon}(t, x)\right|^{2 \sigma+2} \mathrm{e}^{-(2 \sigma+2) Y_{\varepsilon}(x)} \mathrm{d} x \\
& +K_{\varepsilon}\left(1+\left\|v_{0}\right\|_{L^{2}\left(\langle x\rangle^{\delta}\right)}^{a}\right)\left(1+\left\|v_{\varepsilon}\right\|_{C_{T} H^{1}(\langle x\rangle-\delta)}^{3 / 2}\right) \\
& \stackrel{(13)}{\leqslant} c_{\lambda} K_{\varepsilon} \sup _{t \in[0, T]} \int_{\mathbb{R}^{2}}\left|v_{\varepsilon}(t, x)\right|^{2 \sigma+2} \mathrm{e}^{-(2 \sigma+2) Y_{\varepsilon}(x)} \mathrm{d} x+K_{\varepsilon}\left(1+\left\|v_{0}\right\|_{L^{2}\left(\langle x\rangle^{\delta}\right)}^{a}\right)+\frac{1}{2}\left\|v_{\varepsilon}\right\|_{C_{T} H^{1}(\langle x\rangle-\delta)}^{2},
\end{aligned}
$$

where we applied in the last step (13) with $x=\left\|v_{\varepsilon}\right\|_{C_{T} H^{1}(\langle x\rangle-\delta)}^{2}, \theta=\frac{3}{4}, \zeta=1 / 2$ (and changed $a$ and $\left.K_{\varepsilon}\right)$. Subtracting $\frac{1}{2}\left\|v_{\varepsilon}\right\|_{C_{T} H^{1}\left(\langle x\rangle^{-\delta}\right)}$ on both sides and multiplying by 2 gives then

$\left\|v_{\varepsilon}\right\|_{C_{T} H^{1}(\langle x\rangle-\delta)}^{2} \leqslant K_{\varepsilon}\left(1+\left\|v_{0}\right\|_{L^{2}\left(\langle x\rangle^{\delta_{0}}\right)}^{a}\right)+c_{\lambda} K_{\varepsilon} \sup _{t \in[0, T]} \int_{\mathbb{R}^{2}}\left|v_{\varepsilon}\right|^{2 \sigma+2} \mathrm{e}^{-(2 \sigma+2) Y_{\varepsilon}} \mathrm{d} x$.

If $\lambda \leqslant 0$ we have $c_{\lambda} \leqslant 0$ and the last term is non-positive and can be dropped, otherwise consider the case $\sigma<1$. Choose in the following $\kappa>0$ so small that $\sigma+\kappa / 2<1$. Fix further $\bar{\delta} \in\left(0, \delta_{0}\right), \bar{\delta}^{\prime}>0$ such that $\bar{\delta}_{\text {int }}:=\kappa \bar{\delta}-(1-\kappa) \bar{\delta}^{\prime}>0$ and pick finally, without loss of generality, $\delta>0$ so small that we have both $\delta_{\text {int }}:=\frac{\sigma}{\sigma+1}(-\delta)+\frac{1}{\sigma+1} \bar{\delta}_{\text {int }}>0$ and $\delta<1-2 \bar{\delta}$. We have by the Sobolev embedding ( (ii) in lemma 2.1)

$$
H^{\frac{\sigma}{\sigma+1}}\left(\langle x\rangle^{\delta_{\text {int }}}\right) \subseteq L^{2 \sigma+2}\left(\langle x\rangle^{\delta_{\text {int }}}\right) .
$$

Using lemma 2.7 and this continuous embedding we obtain

$$
\left.\sup _{t \in[0, T]} \int_{\mathbb{R}^{2}}\left|v_{\varepsilon}(t, x)\right|^{2 \sigma+2} \mathrm{e}^{-(2 \sigma+2) Y_{\varepsilon}(x)} \mathrm{d} x \stackrel{\text { lemma } 2.7}{\lessgtr} K_{\varepsilon}\left\|v_{\varepsilon}\right\|_{C_{T} L^{2 \sigma+2}\left(\langle x\rangle^{\delta_{\text {int }}}\right)}^{2 \sigma+2} \leqslant K_{\varepsilon}\left\|v_{\varepsilon}\right\|_{C_{T} H^{\frac{\sigma}{\sigma+1}}\left(\langle x\rangle^{\delta_{\text {int }}}\right.}^{2 \sigma+2}\right) .
$$

Recalling that $\delta_{\text {int }}:=\frac{\sigma}{\sigma+1}(-\delta)+\frac{1}{\sigma+1} \bar{\delta}_{\text {int }}$ we can apply interpolation (lemma 2.2) between $H^{1}\left(\langle x\rangle^{-\delta}\right)=\mathcal{B}_{2,2}^{1}\left(\langle x\rangle^{-\delta}\right)$ and $L^{2}\left(\langle x\rangle^{\delta_{\text {int }}}\right)=\mathcal{B}_{2,2}^{0}\left(\langle x\rangle^{\overline{i n t}_{\text {int }}}\right)$. This yields for the right hand side

$$
\begin{aligned}
& K_{\varepsilon}\left\|v_{\varepsilon}\right\|_{C_{T} H^{\frac{\sigma}{\sigma+1}}}^{2 \sigma+2}\left(\langle x\rangle \delta_{\text {int }}\right) \stackrel{\text { lemma } 2.2}{\lessgtr} K_{\varepsilon}\left\|v_{\varepsilon}\right\|_{C_{T} H^{1}(\langle x\rangle-\delta)}^{(2 \sigma+2) \frac{\sigma}{\sigma+1}}\left\|v_{\varepsilon}\right\|_{C_{T} L^{2}\left(\langle x\rangle^{\delta_{\text {int }}}\right)}^{(2 \sigma+2)\left(1-\frac{\sigma}{\sigma+1}\right)} \\
& =K_{\varepsilon}\left\|v_{\varepsilon}\right\|_{C_{T} H^{1}\left(\langle x\rangle^{-\delta}\right)}^{2 \sigma}\left\|v_{\varepsilon}\right\|_{C_{T} L^{2}\left(\langle x\rangle^{\bar{\delta}}\right)^{2}}^{2} .
\end{aligned}
$$

For the second factor we apply once more interpolation to split the weight with exponent $\bar{\delta}_{\text {int }}=\frac{\sigma}{\sigma+1}(-\delta)+\frac{1}{\sigma+1} \bar{\delta}_{\text {int }}$ :

$$
\begin{aligned}
\left\|v_{\varepsilon}\right\|_{C_{T} L^{2}\left(\langle x\rangle^{\bar{\delta}}\right)}^{2} \stackrel{\text { lemma 2.2 }}{\leqslant} C\left\|v_{\varepsilon}\right\|_{C_{T} L^{2}\left(\langle x\rangle-\bar{\delta}^{\prime}\right)}^{2(1-\kappa)}\left\|v_{\varepsilon}\right\|_{C_{T} L^{2}\left(\langle x\rangle^{\bar{\delta}}\right)}^{2 \kappa} \\
\stackrel{\left({ }^{32)} \&\right. \text { lemma 3.1 }}{\leqslant} K_{\varepsilon}\left(1+\left\|v_{0}\right\|_{L^{2}\left(\langle x\rangle^{\left.\delta_{0}\right)}\right.}^{a}\right)\left(1+\left\|v_{\varepsilon}\right\|_{C_{T} H^{1}(\langle x\rangle-\delta)}^{\kappa}\right) .
\end{aligned}
$$

Altogether we obtain

$$
\begin{aligned}
\sup _{t \in[0, T]} \int_{\mathbb{R}^{2}}\left|v_{\varepsilon}(t, x)\right|^{2 \sigma+2} \mathrm{e}^{-(2 \sigma+2) Y_{\varepsilon}(x)} \mathrm{d} x & \leqslant K_{\varepsilon}\left\|v_{\varepsilon}\right\|_{C_{T} H^{1}(\langle x\rangle-\delta)}^{2 \sigma} \\
& \cdot\left(1+\left\|v_{0}\right\|_{L^{2}\left(\langle x\rangle^{\delta}\right)}^{a}\right)\left(1+\left\|v_{\varepsilon}\right\|_{C_{T} H^{1}(\langle x\rangle-\delta)}^{\kappa}\right) \\
& \leqslant K_{\varepsilon}\left(1+\left\|v_{0}\right\|_{L^{2}\left(\langle x\rangle^{\delta}\right)}^{a}\right)\left(1+\left\|v_{\varepsilon}\right\|_{C_{T} H^{1}(\langle x\rangle-\delta)}^{2(\sigma \delta+\kappa / 2)}\right) .
\end{aligned}
$$

Inserting this into (34) and applying (13) (with $x=\left\|v_{\varepsilon}\right\|_{C_{T} H^{1}(\langle x\rangle-\delta)}^{2}, \Theta=\sigma+\kappa / 2, \zeta=\frac{1}{2}$ ) we get 


$$
\begin{aligned}
\left\|v_{\varepsilon}\right\|_{C_{T} H^{1}(\langle x\rangle-\delta)}^{2} & \leqslant K_{\varepsilon}\left(1+\left\|v_{0}\right\|_{L^{2}\left(\langle x\rangle^{\delta_{0}}\right)}^{a}\right)+c_{\lambda} K_{\varepsilon}\left(1+\left\|v_{0}\right\|_{L^{2}\left(\langle x\rangle^{\delta_{0}}\right)}^{a}\right)\left(1+\left\|v_{\varepsilon}\right\|_{C_{T} H^{1}(\langle x\rangle-\delta)}^{2(\sigma+\kappa / 2)}\right) \\
& \stackrel{(13)}{\leqslant} K_{\varepsilon}\left(1+\left\|v_{0}\right\|_{L^{2}\left(\langle x\rangle^{\delta}\right)}^{a}\right)+\frac{1}{2}\left\|v_{\varepsilon}\right\|_{C_{T} H^{1}(\langle x\rangle-\delta)}^{2}
\end{aligned}
$$

(where we changed $a$ and absorbed $c_{\lambda}$ in $K_{\varepsilon}$ in the second line). Subtracting the rightmost term, multiplying by 2 and taking the square root closes the estimate.

Corollary 3.3. If $v_{\varepsilon}$ is a solution to (29) on $[0, T]$ for $T>0$ we have for $\gamma \in[0,1)$ and $\delta<(1-\gamma) \delta_{0}$

$$
\left\|v_{\varepsilon}\right\|_{C_{T} H^{\gamma}\left(\langle x\rangle^{\delta}\right)} \leqslant K_{\varepsilon}\left(1+\left\|v_{0}\right\|_{H^{1}\left(\langle x\rangle^{\left.\delta_{0}\right)}\right.}^{a}\right)
$$

for some deterministic $a>0$.

Proof. Inserting the estimate of proposition 3.2 in lemma 3.1 we obtain for any $\delta_{1} \in\left(0, \delta_{0}\right)$ and some $a>0$

$$
\left\|v_{\varepsilon}\right\|_{C_{T} L^{2}\left(\langle x\rangle^{\delta_{1}}\right)} \leqslant K_{\varepsilon}\left(1+\left\|v_{0}\right\|_{H^{1}\left(\langle x\rangle^{\delta_{0}}\right)}^{a}\right) .
$$

Pick now some $\delta_{1} \in\left(0, \delta_{0}\right)$ such that $\delta<(1-\gamma) \delta_{1}$ and then some $\delta_{2}>0$ such that $\delta=(1-\gamma) \delta_{1}+\gamma\left(-\delta_{2}\right)$. By proposition 3.2 we know that

$$
\left\|v_{\varepsilon}\right\|_{C_{T} H^{1}\left(\langle x\rangle^{-\delta_{2}}\right)} \leqslant K_{\varepsilon}\left(1+\left\|v_{0}\right\|_{H^{1}\left(\langle x\rangle^{\left.\delta_{0}\right)}\right.}^{a}\right) .
$$

The result then follows by interpolating between (35) and (36) via lemma 2.2.

\section{Local existence}

To conclude existence of a solution to (27) a bound in $H^{\gamma}, \gamma>1$ is needed to make sense of the product term $\nabla v_{\varepsilon} \nabla Y_{\varepsilon}$ in the limit $\varepsilon \rightarrow 0$. In [DW18] this was achieved by estimating the $L^{2}$ norm of the time derivative $w_{\varepsilon}=\frac{\mathrm{d}}{\mathrm{d} t} v_{\varepsilon}$, which morally corresponds to bounding the $H^{2}$ norm of $v_{\varepsilon}$. A key role in this estimate is the Brezis-Gallouet inequality [BG80] which is illsuited for our approach based on polynomial weights. In section 5 we show that if $\sigma<1 / 2$ this approach can be modified to yield global existence on $\mathbb{R}^{2}$. We here prove that one has local existence in time provided that one has either $\lambda \leqslant 0$ or $\sigma<1$. Again we work under the assumptions of proposition 2.11 and $T$ is assumed to be smaller than $\tau_{0}$ when $\sigma \geqslant 1$.

Lemma 4.1. Let $v_{\varepsilon}$ be the unique solution to (29) with $\lambda \leqslant 0$ or $\sigma<1$ on $[0, T]$ for $T>0$. We then have for $\delta>0$

$\left\|v_{\varepsilon}\right\|_{L^{\infty}\left(0, T ; H^{2}(\langle x\rangle-\delta)\right)} \leqslant K_{\varepsilon}\left(1+\left\|v_{0}\right\|_{H^{2}\left(\langle x\rangle^{\delta}\right)}^{a}\right) \mathrm{e}^{C T\left\|v_{\varepsilon} \mathrm{e}^{-Y_{\varepsilon}}\right\|_{C_{T} L^{\infty}\left(\mathbb{R}^{2}\right)}^{2 \sigma}\left(1+|\log (\varepsilon)|^{a}\right),}$

for some deterministic $a>0$.

Proof. We consider as in [DW18] the quantity $w_{\varepsilon}=\partial_{t} v_{\varepsilon}$ which satisfies the equation $\imath \partial_{t} w_{\varepsilon}=\Delta w_{\varepsilon}+w_{\varepsilon}: \widetilde{\nabla Y_{\varepsilon}^{2}}:-2 \nabla w_{\varepsilon} \nabla Y_{\varepsilon}+\lambda\left|v_{\varepsilon} \mathrm{e}^{-Y_{\varepsilon}}\right|^{2 \sigma} w_{\varepsilon}+2 \sigma \lambda v_{\varepsilon}\left|v_{\varepsilon}\right|^{2 \sigma-2} \operatorname{Re}\left(w_{\varepsilon} \bar{v}_{\varepsilon}\right) \mathrm{e}^{-2 \sigma Y_{\varepsilon}}$ 
and whose mass evolves like

$$
\begin{aligned}
& \frac{1}{2} \frac{\mathrm{d}}{\mathrm{d} t}\left(\int_{\mathbb{R}^{2}}\left|w_{\varepsilon}(\cdot, x)\right|^{2} \mathrm{e}^{-2 Y_{\varepsilon}(x)} \mathrm{d} x\right)(t)=\operatorname{Im}\left(\int_{\mathbb{R}^{2}} \imath \partial_{t} w_{\varepsilon}(t, x) \cdot \bar{w}_{\varepsilon}(t, x) \mathrm{d} x\right) \\
& \stackrel{(37)}{=} 2 \sigma \lambda \int_{\mathbb{R}^{2}} \operatorname{Im}\left(\bar{w}_{\varepsilon}(t, x) v_{\varepsilon}(t, x)\right) \operatorname{Re}\left(w_{\varepsilon}(t, x) \bar{v}_{\varepsilon}(t, x)\right)\left|v_{\varepsilon}(t, x)\right|^{2 \sigma-2} \mathrm{e}^{-(2 \sigma+2) Y_{\varepsilon}(x)} \mathrm{d} x \\
& \leqslant C\left\|v_{\varepsilon} \mathrm{e}^{-Y_{\varepsilon}}\right\|_{L^{\infty}}^{2 \sigma} \int_{\mathbb{R}^{2}}\left|w_{\varepsilon}(t, x)\right|^{2} \mathrm{e}^{-2 Y_{\varepsilon}(x)} \mathrm{d} x .
\end{aligned}
$$

Gronwall's lemma provides then

$\int_{\mathbb{R}^{2}}\left|w_{\varepsilon}(t, x)\right|^{2} \mathrm{e}^{-2 Y_{\varepsilon}(x)} \mathrm{d} x \leqslant \int_{\mathbb{R}^{2}}\left|w_{\varepsilon}(0, x)\right|^{2} \mathrm{e}^{-2 Y_{\varepsilon}(x)} \mathrm{d} x \cdot \mathrm{e}^{C T\left\|v_{\varepsilon} \mathrm{e}^{-Y_{\varepsilon}}\right\|_{T_{T} L^{\infty}\left(\mathbb{R}^{2}\right)}^{2 \sigma}}$

Observe that, by definition of $w_{\varepsilon}$ and the fact that $v_{\varepsilon}$ solves (27),

$$
\imath w_{\varepsilon}=\Delta v_{\varepsilon}+v_{\varepsilon}: \widetilde{\nabla Y_{\varepsilon}^{2}}:-2 \nabla v_{\varepsilon} \nabla Y_{\varepsilon}+\lambda\left|v_{\varepsilon}\right|^{2 \sigma} v_{\varepsilon} \mathrm{e}^{-2 \sigma Y_{\varepsilon}} .
$$

Using this identity at $t=0$, where $v_{\varepsilon}(0)=v_{0}$, we obtain for $q$ close enough to 2 such that $q^{\prime}$ with $\frac{1}{2}=\frac{1}{q}+\frac{1}{q^{\prime}}$ satisfies $q^{\prime} \delta_{0}>4$ by (11) and Hölder's inequality

$$
\begin{aligned}
\left\|w_{\varepsilon}(0)\right\|_{L^{2}\left(\langle x\rangle^{\frac{\delta_{0}}{2}}\right)} & \leqslant C\left\|v_{0}\right\|_{H^{2}\left(\langle x\rangle^{\frac{\delta_{0}}{2}}\right)}+\left\|v_{0}\right\|_{L^{q}\left(\langle x\rangle^{\delta_{0}}\right)}\left\|\widetilde{\nabla Y_{\varepsilon}^{2}:}\right\|_{L^{q^{\prime}}\left(\langle x\rangle^{-\delta_{0} / 2}\right)} \\
& +2\left\|\nabla v_{0}\right\|_{L^{q}\left(\langle x\rangle^{\delta_{0}}\right)}\left\|\nabla Y_{\varepsilon}\right\|_{\left.L^{q^{\prime}(\langle x\rangle}-\delta_{0} / 2\right)}+\lambda\left\|\left.|| v_{0}\right|^{2 \sigma+1}\right\|_{L^{q}\left(\langle x\rangle^{\delta_{0}}\right)}\left\|\mathrm{e}^{-2 \sigma Y_{\varepsilon}}\right\|_{L^{q^{\prime}}\left(\langle x\rangle^{-\delta_{0} / 2}\right)} \\
& \leqslant K_{\varepsilon}\left(1+\left\|v_{0}\right\|_{H^{2}\left(\langle x\rangle^{\delta_{0}}\right)}^{a}\right) \cdot\left(1+|\log \varepsilon|^{a}\right) .
\end{aligned}
$$

As usual $a>0$ denotes as a changing deterministic constant. In the last step (41) we made use of lemma 2.10 and Sobolev embedding ( (ii) in lemma 2.1) which implies $\left\|v_{0}\right\|_{L^{q}\left(\langle x\rangle^{\delta}\right)}$, $\left\|\nabla v_{0}\right\|_{L^{q}\left(\langle x\rangle^{\delta}\right)} \leqslant C\left\|v_{0}\right\|_{H^{2}\left(\langle x\rangle^{\left.\delta_{0}\right)}\right.}$ and

$\left\|\left|v_{0}\right|^{2 \sigma+1}\right\|_{L^{q}\left(\langle x\rangle^{\delta_{0}}\right)}=\left\|v_{0}\right\|_{L^{q(2 \sigma+1)}\left(\langle x\rangle^{\delta_{0} /(2 \sigma+1)}\right)}^{2 \sigma+1} \stackrel{\text { lemma 2.1 (ii) }}{\lessgtr} C\left\|v_{0}\right\|_{H^{1}\left(\langle x\rangle^{\delta_{0} /(2 \sigma+1)}\right)}^{2 \sigma+1} \leqslant C\left\|v_{0}\right\|_{H^{2}\left(\langle x\rangle^{\delta_{0}}\right)}^{2 \sigma+1}$.

We can now bound the right hand side of (39) via lemma 2.7 and (41) and end up with:

$$
\int_{\mathbb{R}^{2}}\left|w_{\varepsilon}(t)\right|^{2} \mathrm{e}^{-2 Y_{\varepsilon}} \leqslant K_{\varepsilon}\left(1+\left\|v_{0}\right\|_{H^{2}\left(\langle x\rangle^{\delta_{0}}\right)}^{a}\right) \cdot\left(1+|\log \varepsilon|^{a}\right) \cdot \mathrm{e}^{C T\left\|v_{\varepsilon} \mathrm{e}^{-Y_{\varepsilon}}\right\|_{C_{T} L^{\infty}\left(\mathbb{R}^{2}\right)}^{2 \sigma}} .
$$

Consequently, our task can now be reduced to show an inequality of the form

$\left\|v_{\varepsilon}\right\|_{C_{T} H^{2}\left(\langle x\rangle^{-\delta}\right)} \leqslant C\left\|w_{\varepsilon} \cdot \mathrm{e}^{-2 Y_{\varepsilon}}\right\|_{C_{T} L^{2}}+K_{\varepsilon}\left(1+\left\|v_{0}\right\|_{H^{2}\left(\langle x\rangle^{\delta_{0}}\right)}^{a}\right)\left(1+|\log \varepsilon|^{a}\right)$.

To show (42) we use that by reshaping (40) and Hölder's inequality

$$
\begin{aligned}
& \left\|\Delta v_{\varepsilon}(t)\right\|_{L^{2}\left(\langle x\rangle^{-\delta}\right)} \leqslant\left\|w_{\varepsilon}(t)\right\|_{L^{2}\left(\langle x\rangle^{-\delta}\right)}+\left\|v_{\varepsilon}(t)\right\|_{L^{q}\left(\langle x\rangle^{-3 \delta / 4}\right)}\left\|: \widetilde{\nabla Y_{\varepsilon}^{2}:}\right\|_{L^{q^{\prime}}\left(\langle x\rangle^{-\delta / 4}\right)} \\
& +2\left\|\nabla v_{\varepsilon}(t)\right\|_{L^{q}\left(\langle x\rangle^{-3 \delta / 4}\right)}\left\|\nabla Y_{\varepsilon}\right\|_{L^{q^{\prime}}\left(\langle x\rangle^{-\delta / 4}\right)}+\left\|\left.v_{\varepsilon}(t)\right|^{2 \sigma+1}\right\|_{L^{q}\left(\langle x\rangle^{-3 \delta / 4}\right)}\left\|\mathrm{e}^{-(2 \sigma+1) Y_{\varepsilon}}\right\|_{L^{q^{\prime}}\left(\langle x\rangle^{-\delta / 4}\right)},
\end{aligned}
$$

where $q \in(2,4)$ is now small enough such that $q^{\prime}$ with $\frac{1}{2}=\frac{1}{q}+\frac{1}{q^{\prime}}$ satisfies $q^{\prime} \cdot \delta>8$. By Sobolev embedding ( ( ii) of lemma 2.1) we have for any $r \in[1, \infty)$ and $\delta^{\prime}>0$

$$
\left\|v_{\varepsilon}(t)\right\|_{L^{r}\left(\langle x\rangle^{-\delta^{\prime}}\right)} \leqslant C \cdot\left\|v_{\varepsilon}(t)\right\|_{H^{1}\left(\langle x\rangle-\delta^{\prime}\right)} \stackrel{\text { proposition } 3.2}{\leqslant} K_{\varepsilon} \cdot\left(1+\left\|v_{0}\right\|_{H^{1}\left(\langle x\rangle^{\delta_{0}}\right)}^{a}\right)
$$


so that in particular $\left\|v_{\varepsilon}(t)\right\|_{L^{q}(\langle x\rangle-3 \delta / 4)} \leqslant K_{\varepsilon} \cdot\left(1+\left\|v_{0}\right\|_{H^{1}\left(\left\langle x \delta^{\left.\delta_{0}\right)}\right.\right.}^{a}\right)$ and $\left\|\left|v_{\varepsilon}(t)\right|^{2 \sigma+1}\right\|_{L^{q}(\langle x\rangle-3 \delta / 4)}=$ $\left\|\left|v_{\varepsilon}(t)\right|\right\|_{L^{q \cdot(2 \sigma+1)}\left(\langle x\rangle^{-3 \delta / 4(2 \sigma+1)}\right)}^{2 \sigma+1} \leqslant K_{\varepsilon} \cdot\left(1+\left\|v_{0}\right\|_{H^{1}\left(\langle x\rangle^{\delta_{0}}\right)}^{a}\right)$. The factor $\left\|\nabla v_{\varepsilon}(t)\right\|_{L^{q}\left(\langle x\rangle^{-3 \delta / 4}\right)}$ in

(43) can once more be bounded by Sobolev embedding and interpolation (lemmas 2.1 and 2.2)

$\left\|\nabla v_{\varepsilon}(t)\right\|_{L^{q}\left(\langle x\rangle^{-3 \delta / 4}\right)}\|\stackrel{\text { lemma } 2.1}{\leqslant} C\| v_{\varepsilon}(t)\left\|_{H^{3 / 2}\left(\langle x\rangle^{-3 \delta / 4}\right)} \stackrel{\text { lemma } 2.2}{\leqslant} C\right\| v_{\varepsilon}(t)\left\|_{H^{1}\left(\langle x\rangle^{-\delta / 2}\right)}^{\frac{1}{2}}\right\| v_{\varepsilon}(t) \|_{H^{2}\left(\langle x\rangle^{-\delta}\right)^{\frac{1}{2}}}^{{ }^{2}}$,

where we used $q<4$ for the embedding $L^{q}\left(\langle x\rangle^{-3 \delta / 4}\right) \subseteq H^{3 / 2}\left(\langle x\rangle^{-3 \delta / 4}\right)$. Applying proposition 3.2 we therefore obtain in total for some $a>0$

$\left\|\Delta v_{\varepsilon}(t)\right\|_{L^{2}\left(\langle x\rangle^{-\delta}\right)} \leqslant\left\|w_{\varepsilon}(t)\right\|_{L^{2}\left(\langle x\rangle^{-\delta}\right)}+K_{\varepsilon}\left(1+\left\|v_{0}\right\|_{H^{2}\left(\langle x\rangle^{\delta}\right)}^{a}\right)\left(1+|\log \varepsilon|^{a}\right)\left(1+\left\|v_{\varepsilon}(t)\right\|_{H^{2}\left(\langle x\rangle^{-\delta}\right)}^{1 / 2}\right)$.

It is easy to see via (8) that $\|g\|_{H^{2}\left(\langle x\rangle^{-\delta}\right)} \leqslant C\left(\|g\|_{H^{1}\left(\langle x\rangle^{-\delta}\right)}+\|\Delta g\|_{L^{2}\left(\langle x\rangle^{-\delta}\right)}\right)$ so that we obtain $\left\|v_{\varepsilon}(t)\right\|_{H^{2}\left(\langle x\rangle^{-\delta}\right)} \leqslant C\left\|w_{\varepsilon}(t)\right\|_{L^{2}(\langle x\rangle-\delta)}+K_{\varepsilon}\left(1+\left\|v_{0}\right\|_{H^{2}\left(\langle x\rangle^{\delta}\right)}^{a}\right)\left(1+|\log \varepsilon|^{a}\right)\left(1+\left\|v_{\varepsilon}(t)\right\|_{H^{2}(\langle x\rangle-\delta)}^{1 / 2}\right)$.

Applying (13) (with $x=\left\|v_{\varepsilon}(t)\right\|_{H^{2}\langle x\rangle^{-\delta}}, \Theta=\frac{1}{2}, \zeta=\frac{1}{2}$ ) this can be reshaped to $\left\|v_{\varepsilon}(t)\right\|_{H^{2}(\langle x\rangle-\delta)} \leqslant C\left\|w_{\varepsilon}(t)\right\|_{L^{2}(\langle x\rangle-\delta)}+K_{\varepsilon}\left(1+\left\|v_{0}\right\|_{H^{2}\left(\langle x\rangle^{\delta}\right)}^{a}\right)\left(1+|\log \varepsilon|^{a}\right)+\frac{1}{2}\left\|v_{\varepsilon}(t)\right\|_{H^{2}\langle x\rangle-\delta}$.

Subtracting the last term and multiplying both sides by 2 yields (42) and closes the proof.

Remark 4.2. This proof is not completely rigorous since we do not know that the solution $v_{\varepsilon}$ is sufficiently smooth to perform the above computations, in particular to justify (38). The remedy is classical. We smooth the initial data and take $v_{\varepsilon}^{N}(0)=\rho_{\frac{1}{N}} * v_{0}$, replace the nonlinear term by $\left(|v|^{2}+1 / n\right)^{\sigma} v$ and as in the proof of proposition 2.11 , truncate the noise. Then by [Caz03], theorem 5.4.1, we know that the solution is continuous with values in $H^{4}$ and thus $C^{2}$ with values in $L^{2}$ so that its time derivative is $C^{1}$ with values in $L^{2}$. This is clear in the case $\sigma<1$. In the case $\lambda \leqslant 0$, we first write the equation satisfied by $v_{\varepsilon, N, n} \mathrm{e}^{-Y_{\varepsilon, N}}$, where $v_{\varepsilon, N, n}$ is the solution of equation (A.9) with the modified nonlinear term. It is similar to (A.1) with an additional potential $: \nabla Y_{\varepsilon}^{2}:-\nabla Y_{\varepsilon, N}^{2}$ and the modified nonlinear term. By [Caz03], theorem 5.4.1, this equation has a smooth solution and this shows that $v_{\varepsilon, N, n}$ has the same smoothness. The above computations are done on this modified equation, a bound uniform in $n, N$ is obtained. The results follow by letting $n, N \rightarrow \infty$.

This estimate is sufficient to prove local existence of a solution $v$ to (27) on some random interval $[0, \tau]$. We follow [DW18] in the consideration of the differences of the dyadic sequence. Recall that the notation $K_{k}$ in the following stands for a random constant of the form $K_{k}=K_{2^{-k}} K_{2^{-k-1}}$ that can be bounded almost surely in $k$. To derive the latter property we will always use the polynomial convergence rates from (22)-(24) together with the Borell-Cantelli lemma, for instance

$$
\left\|Y_{2^{-k}}-Y_{2^{-k-1}}\right\|_{\mathcal{C}^{\alpha}\left(\langle x\rangle^{-\delta}\right)} \leqslant\left\|Y_{2^{-k}}-Y\right\|_{\mathcal{C}^{\alpha}\left(\langle x\rangle^{-\delta}\right)}+\left\|Y-Y_{2^{-k-1}}\right\|_{\mathcal{C}^{\alpha}\left(\langle x\rangle^{-\delta}\right)} \leqslant K_{k}
$$

for $\alpha \in(0,1)$ and $\delta>0$.

Lemma 4.3. Let $v_{2^{-k}}$ be the unique solution to (29) on $[0, T]$ for $T>0$ with $\varepsilon=2^{-k}$ and $\lambda \leqslant 0$ or $\sigma<1$. We then have for $\gamma \in(0,2), \delta<\left(1-\frac{\gamma}{2}\right) \delta_{0}$, 


$$
\begin{aligned}
& \left\|v_{2-k}-v_{2-k-1}\right\|_{C_{T} H^{\gamma}\left(\langle x\rangle^{\delta}\right)} \\
& \leqslant K_{k} 2^{-k \kappa}\left(1+\left\|v_{0}\right\|_{H^{2}\left(\langle x\rangle^{\delta_{0}}\right)}^{a}\right) \mathrm{e}^{C T\left(\| v_{2}-k\right.} \mathrm{e}^{-Y_{2}-k}\left\|_{C_{T^{2}}}^{2 \sigma}+\right\| v_{2-k-1} \mathrm{e}^{-Y_{2}-k-1} \|_{\left.C_{T} L^{\infty}\right)}^{2 \sigma},
\end{aligned}
$$

for some $\kappa>0$ and $a>0$, where the sequence of random constants $K_{k}$ is bounded almost surely.

Proof. The difference $r_{k}=v_{2-k}-v_{2^{-k-1}}$ satisfies the equation

$$
\begin{aligned}
\imath \partial_{t} r_{k}= & \Delta r_{k}+r_{k}: \widetilde{\nabla Y_{2^{-k-1}}^{2}}:-2 \nabla r_{k} \nabla Y_{2^{-k-1}}+v_{2^{-k}}\left(: \widetilde{\nabla Y_{2^{-k-1}}^{2}}:-\widetilde{\nabla Y_{2^{-k}}^{2}}:\right) \\
& -2 \nabla v_{2^{-k}}\left(\nabla Y_{2^{-k-1}}-\nabla Y_{2^{-k}}\right)+\lambda\left(\left|v_{2^{-k}} \mathrm{e}^{-Y_{2-k}}\right|^{2 \sigma} v_{2^{-k}}-\left|v_{2^{-k-1}} \mathrm{e}^{-Y_{2-k-1}}\right|^{2 \sigma} v_{2^{-k-1}}\right) .
\end{aligned}
$$

Since $v_{2-k-1} \mathrm{e}^{-Y_{2-k-1}(x)}$ and $v_{2-k} \mathrm{e}^{-Y_{2-k-1}(x)}$ are in $C_{T} H^{\gamma}\left(\langle x\rangle^{\delta}\right)$ for $\gamma \in(1,2)$ and $\delta<\left(1-\frac{\gamma}{2}\right) \delta_{0}$, we can check that that $\partial_{t} r_{k} \mathrm{e}^{-Y_{2-k-1}} \in C_{T} H^{\gamma-2}\left(\langle x\rangle^{\delta}\right)$. This latter space is a subspace of the dual of $C_{T} H^{\gamma}\left(\langle x\rangle^{\delta}\right)$ so that we may write

$$
\frac{1}{2} \frac{\mathrm{d}}{\mathrm{d} t}\left(\int_{\mathbb{R}^{2}}\left|r_{k}(t, x)\right|^{2} \mathrm{e}^{-2 Y_{2-k-1}(x)} \mathrm{d} x\right)(t)=\left\langle\partial_{t} r_{k} \mathrm{e}^{-Y_{2-k-1}(x)}, r_{k} \mathrm{e}^{-Y_{2}-k-1}(x)\right\rangle
$$

when the brackets stands for the duality product. It follows that the 'mass' of $r_{k}$ evolves according to

$$
\begin{aligned}
& \frac{1}{2} \frac{\mathrm{d}}{\mathrm{d} t}\left(\int_{\mathbb{R}^{2}}\left|r_{k}(t, x)\right|^{2} \mathrm{e}^{-2 Y_{2-k-1}(x)} \mathrm{d} x\right)(t) \\
& =\operatorname{Im}\left\{\int _ { \mathbb { R } ^ { 2 } } \left(v_{2^{-k}}(t, x)\left(: \nabla \widehat{Y_{2^{-k-1}}^{2}}(x):-: \widetilde{\nabla Y_{2-k}^{2}(x)}\right): \bar{r}_{k}(t, x) \mathrm{e}^{-2 Y_{2-k-1}(x)}\right.\right. \\
& \quad-2 \nabla v_{2^{-k}}(t, x)\left(\nabla Y_{2^{-k-1}}(x)-\nabla Y_{2^{-k}}(x)\right) \bar{r}_{k}(t, x) \mathrm{e}^{-2 Y_{2-k-1}(x)} \\
& \quad+\lambda\left(\left|v_{2^{-k}}(t, x) \mathrm{e}^{-Y_{2-k}(x)}\right|^{2 \sigma} v_{2^{-k}}(t, x)\right. \\
& \left.\left.\left.\quad-\left|v_{2^{-k-1}}(x) \mathrm{e}^{-Y_{2-k-1}(x)}\right|^{2 \sigma} v_{2^{-k-1}}(t, x)\right) \bar{r}_{k}(t, x) \mathrm{e}^{-2 Y_{2-k-1}(x)}\right) \mathrm{d} x\right\} .
\end{aligned}
$$

Via (iii), (iv) of lemma 2.1 (Duality and Multiplication bound) and using interpolation between the bound in corollary 3.3 and lemma 4.1 we can estimate the first two terms on the right hand side, up to a constant, by

$$
\begin{aligned}
& \left\|v_{2-k} \bar{r}_{k} \mathrm{e}^{-2 Y_{2-k-1}}\right\|_{C_{T} \mathcal{B}_{1,1}^{\frac{1}{2}-\kappa^{\prime \prime}}\left(\langle x\rangle^{\delta^{\prime}}\right)}\left\|: \widetilde{\nabla Y_{2^{-k-1}}^{2}}:-\widetilde{\nabla Y_{2^{-k}}^{2}}:\right\|_{\mathcal{C}^{-\frac{1}{2}+\kappa^{\prime \prime}}\left(\langle x\rangle-\delta^{\prime}\right)} \\
& +\left\|\nabla v_{2-k} \bar{r}_{k} \mathrm{e}^{-2 Y_{2-k-1}}\right\|_{C_{T} \mathcal{B}_{1,1}^{\frac{1}{2}-\kappa^{\prime \prime}}\left(\langle x\rangle^{\delta^{\prime}}\right)}\left\|\left(\nabla Y_{2^{-k-1}}-\nabla Y_{2-k}\right)\right\|_{\mathcal{C}^{-\frac{1}{2}+\kappa^{\prime \prime}}\left(\langle x\rangle^{-\delta^{\prime}}\right)} \\
& \stackrel{\text { lemma } 2.8}{\leqslant} K_{k}\left(\left\|v_{2-k}\right\|_{C_{T} H^{\frac{3}{2}}\left(\langle x\rangle^{\delta^{\prime}}\right)}^{2}+\left\|v_{2^{-k-1}}\right\|_{C_{T} H^{\frac{3}{2}}\left(\langle x\rangle^{\delta^{\prime}}\right)}^{2}\right) 2^{-k \kappa^{\prime}}
\end{aligned}
$$

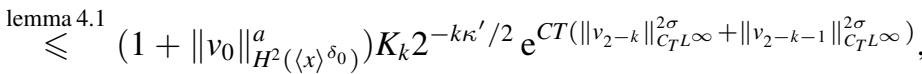

for $\kappa^{\prime}, \kappa^{\prime \prime} \in(0,1 / 2), \delta^{\prime} \in\left(0, \delta_{0} / 4\right)$ and some $a>0$ that changes from line to line. In the last step we used that $2^{-k \kappa^{\prime}} \cdot\left|\log \left(2^{-k}\right)\right|^{a} \leqslant C 2^{-k \kappa^{\prime} / 2}$ to cancel the logarithmic divergence arising from the application of lemma 4.1. Up to a term $K_{k} 2^{-k \kappa}\left(1+\left\|v_{0}\right\|_{H^{1}\left(\langle x\rangle^{\delta_{0}}\right)}^{a}\right)$ we can reshape the third term of the inequality above as 


$$
\begin{aligned}
\operatorname{Im}\left\{\int _ { \mathbb { R } ^ { 2 } } \lambda \left(\left|v_{2^{-k}}(t, x) \mathrm{e}^{-Y_{2-k}(x)}\right|^{2 \sigma} v_{2^{-k}}(x) \mathrm{e}^{-Y_{2-k}(x)}\right.\right. \\
\left.\left.\quad-\left|v_{2^{-k-1}}(t, x) \mathrm{e}^{-Y_{2-k-1}(x)}\right|^{2 \sigma} v_{2^{-k-1}}(x) \mathrm{e}^{-Y_{2-k-1}(x)}\right) \bar{r}_{k}(t, x) \mathrm{e}^{-Y_{2-k-1}(x)} \mathrm{d} x\right\} .
\end{aligned}
$$

Recall that for $x,\left.y \in \mathbb{C}|| x\right|^{2 \sigma} x-|y|^{2 \sigma} y\left|\leqslant C\left(|x|^{2 \sigma}+|y|^{2 \sigma}\right)\right| x-y \mid$ (see for example [Caz03, p 86]) so that we obtain the upper bound

$$
\begin{aligned}
& \int_{\mathbb{R}^{2}}\left(\left|v_{2^{-k}}(t, x) \mathrm{e}^{-Y_{2-k}(x)}\right|^{2 \sigma}+\left|v_{2^{-k-1}}(t, x) \mathrm{e}^{-Y_{2-k-1}(x)}\right|^{2 \sigma}\right) \\
& \cdot\left|v_{2^{-k}}(t, x) \mathrm{e}^{-Y_{2-k}(x)}-v_{2^{-k-1}}(t, x) \mathrm{e}^{-Y_{2-k-1}(x)}\right|\left|r_{k}(t, x)\right| \mathrm{e}^{-Y_{2-k-1}(x)} \mathrm{d} x .
\end{aligned}
$$

Applying (23), corollary 3.3 and Sobolev embedding ( (ii) of lemma 2.1), the above quantity is bounded by the sum of a term $K_{k}\left(1+\left\|v_{0}\right\|_{H^{1}\left(\langle x\rangle \delta_{0}\right)}^{a}\right) 2^{-k \kappa}$ and of:

$$
\begin{aligned}
& \int_{\mathbb{R}^{2}}\left(\left|v_{2^{-k}}(t, x) \mathrm{e}^{-Y_{2-k}(x)}\right|^{2 \sigma}+\left|v_{2^{-k-1}}(t, x) \mathrm{e}^{-Y_{2-k-1}(x)}\right|^{2 \sigma}\right)\left|r_{k}(t, x)\right|^{2} \mathrm{e}^{-2 Y_{2-k-1}(x)} \mathrm{d} x \\
& \leqslant\left(\left\|v_{2^{-k}} \mathrm{e}^{-Y_{2-k}}\right\|_{C_{T} L^{\infty}}^{2 \sigma}+\left\|v_{2-k-1} \mathrm{e}^{-Y_{2-k-1}}\right\|_{C_{T} L^{\infty}}^{2 \sigma}\right) \int_{\mathbb{R}^{2}}\left|r_{k}(t, x)\right|^{2} \mathrm{e}^{-2 Y_{2-k-1}(x)} \mathrm{d} x .
\end{aligned}
$$

Gathering all these estimates yields

$$
\begin{aligned}
& \frac{\mathrm{d}}{\mathrm{d} t}\left(\int_{\mathbb{R}^{2}}\left|r_{k}(\cdot, x)\right|^{2} \mathrm{e}^{-2 Y_{2-k-1}(x)} \mathrm{d} x\right)(t) \leqslant K_{k} 2^{-k \kappa} \mathrm{e}^{C T\left(\left\|v_{2-k}\right\|_{C_{T} L^{\infty}}^{2 \sigma}+\left\|v_{2-k-1}\right\|_{C_{T} L^{\infty}}^{2 \sigma}\right)} \\
& +C\left(\left\|v_{2-k} \mathrm{e}^{-Y_{2}-k}\right\|_{C_{T} L^{\infty}}^{2 \sigma}+\left\|v_{2^{-k-1}} \mathrm{e}^{-Y_{2-k-1}}\right\|_{C_{T} L^{\infty}}^{2 \sigma}\right) \int_{\mathbb{R}^{2}}\left|r_{k}(t, x)\right|^{2} \mathrm{e}^{-2 Y_{2-k-1}(x)} \mathrm{d} x,
\end{aligned}
$$

for some $\kappa>0$. Application of Gronwall's lemma gives together with lemma 2.7

$$
\begin{aligned}
& \left\|v_{2-k}-v_{2-k-1}\right\|_{C_{T} L^{2}\left(\langle x\rangle^{-\delta}\right)} \\
& \leqslant K_{k} 2^{-k \kappa}\left(1+\left\|v_{0}\right\|_{H^{2}\left(\left\langle x \delta_{0}\right)\right.}\right) \mathrm{e}^{C T\left(\left\|v_{2-k} \mathrm{e}^{-Y_{2}-k}\right\|_{C_{T} L^{\infty}}^{2 \sigma}+\left\|v_{2-k-1} \mathrm{e}^{-Y_{2-k-1}}\right\|_{C_{T} L^{\infty}}^{2 \sigma}\right)}
\end{aligned}
$$

for any $\delta>0$. The desired estimate now follows by interpolation with corollary 3.3 and lemma 4.1.

We are now in the position to prove local existence.

Theorem 4.4. Let $\delta_{0} \in\left(0, \frac{1}{2}\right)$ and consider $v_{0}$ with $v_{0} \in H^{2}\left(\langle x\rangle^{\delta_{0}}\right)$ almost surely. There is a random time $\tau>0$ (depending on $v_{0}$ ) such that

$$
\imath \partial_{t} v=\Delta v+v\left(: \nabla Y^{2}:-\varphi * \xi\right)-2 \nabla v \nabla Y+\lambda|v|^{2 \sigma} v \mathrm{e}^{-2 \sigma Y}, v(0)=v_{0}
$$

with $\lambda \leqslant 0$ or $\sigma<1$ has almost surely a (unique) solution $v \in C_{\tau} H^{\gamma}\left(\langle x\rangle^{\delta}\right)$ on $[0, \tau]$ for any $\gamma \in(1,2)$ and $\delta<\left(1-\frac{\gamma}{2}\right) \delta_{0}$. The random variable $\left\|v_{\varepsilon}-v\right\|_{C_{\tau} H^{\gamma}\left(\langle x\rangle^{\delta}\right)}$ converges to 0 in probability.

Proof. Let $M_{T}^{N}:=\sup _{k \leqslant N}\left\|v_{2^{-k}}\right\|_{C_{T} H^{\gamma}\left(\langle x\rangle^{\delta}\right)}$. By (18), we have:

$$
\sup _{x \in[-k, k]^{2}}\left|\mathrm{e}^{-Y_{2-k}(x)}\right|\langle k\rangle^{-\delta} \leqslant \sup _{x \in[-k-1, k+1]^{2}}\left|\mathrm{e}^{-Y(x)}\right|\langle k\rangle^{-\delta} .
$$


Therefore, using corollary 2.6

$$
\left\|\mathrm{e}^{-Y_{2-k}}\langle x\rangle^{-\delta}\right\|_{L^{\infty}\left(\mathbb{R}^{2}\right)} \leqslant \tilde{K}
$$

where $K$ is random with all moments finite. Then, by Sobolev embedding:

$$
\left\|v_{2-k} \mathrm{e}^{-Y_{2-k}}\right\|_{C_{T} L^{\infty}\left(\mathbb{R}^{2}\right)} \leqslant \tilde{K}\left\|v_{2^{-k}}\langle x\rangle^{\delta}\right\|_{C_{T} L^{\infty}\left(\mathbb{R}^{2}\right)} \leqslant c \tilde{K}\left\|v_{2^{-k}}\right\|_{C_{T} H^{\gamma}\left(\langle x\rangle^{\delta}\right)} \leqslant c \tilde{K} M_{T}^{N} .
$$

We then have summing the estimate in lemma 4.3 and using the above to bound the exponential term:

$$
\begin{aligned}
M_{T}^{N} & \leqslant\left\|v_{1}\right\|_{C_{T} H^{\gamma}\left(\langle x\rangle^{\delta}\right)}+\sum_{k=1}^{\infty} K_{k} 2^{-k \kappa}\left(1+\left\|v_{0}\right\|_{H^{2}\left(\langle x\rangle^{\delta_{0}}\right)}^{a}\right) \mathrm{e}^{C \tilde{K} T\left(M_{T}^{N}\right)^{2 \sigma}} \\
& \leqslant K\left(1+\left\|v_{0}\right\|_{H^{2}\left(\langle x\rangle^{\delta}\right)}^{a}\right) \mathrm{e}^{C \tilde{K} T\left(M_{T}^{N}\right)^{2 \sigma}}
\end{aligned}
$$

where the random constant $K$ is finite almost surely and moreover, by Minkowski's inequality, in any $L^{p}(\mathbb{P}), p \in[1, \infty)$. We choose $K$ without loss of generality positive almost everywhere. (44) already shows that there is a random time $\tau$, independent of $N$ such that for any $N \in \mathbb{N}$

$$
M_{\tau}^{N} \leqslant 3 K\left(1+\left\|v_{0}\right\|_{H^{2}\left(\langle x\rangle^{\delta}\right)}^{a}\right)
$$

almost surely and that one might take $\tau$ as the solution of

$$
\left.\left.K\left(1+\left\|v_{0}\right\|_{H^{2}\left(\left\langle x \delta^{\delta_{0}}\right)\right.}^{a}\right) \mathrm{e}^{C \tilde{K} \tau\left(3 K\left(1+\left\|v_{0}\right\|_{H^{2}(\langle x\rangle}^{a} \delta_{0}\right)\right.}\right)\right)^{2 \sigma}=2 K\left(1+\left\|v_{0}\right\|_{H^{2}\left(\langle x\rangle^{\left.\delta_{0}\right)}\right.}^{a}\right) .
$$

Indeed, suppose there is a subset $A$ of the considered probability space with positive probability such that on this set

$$
M_{\tau}^{N}>3 K\left(1+\left\|v_{0}\right\|_{H^{2}\left(\langle x\rangle^{\delta}\right)}^{a}\right)
$$

for $\tau$ as in (46). Note, that $T \mapsto M_{T}^{N}$ is a continuous function and that, due to (44), $M_{0}^{N} \leqslant K\left(1+\left\|v_{0}\right\|_{C_{T} H^{2}\left(\langle x\rangle^{\delta}\right)}^{a}\right)$. If (47) was true, the intermediate value theorem would imply the existence of a (random) time $\tau^{*} \leqslant \tau$ such that on $A$

$$
M_{\tau^{*}}^{N}=3 K\left(1+\left\|v_{0}\right\|_{H^{2}\left(\langle x\rangle^{\delta}\right)}^{a}\right) .
$$

But then, again on $A$,

$$
\begin{aligned}
3 K\left(1+\left\|v_{0}\right\|_{H^{2}\left(\langle x\rangle^{\delta}\right)}^{a}\right) & =M_{\tau^{*}}^{N} \stackrel{(44)}{\leqslant} K\left(1+\left\|v_{0}\right\|_{H^{2}\left(\langle x\rangle^{\delta}\right)}^{a}\right) \mathrm{e}^{C \tilde{K} \tau^{*}\left(M_{\tau^{*}}^{N}\right)^{2 \sigma}} \\
\stackrel{\text { definition of } \tau^{*}}{\leqslant} & K\left(1+\left\|v_{0}\right\|_{H^{2}\left(\langle x\rangle^{\delta}\right)}^{a}\right) \mathrm{e}^{C \tilde{K} \tau\left(3 K\left(1+\left\|v_{0}\right\|_{C_{\tau} H^{2}(\langle x\rangle}^{a}\right)\right)^{2 \sigma}} \stackrel{(46)}{=} 2 K\left(1+\left\|v_{0}\right\|_{H^{2}\left(\langle x\rangle^{\delta}\right)}^{a}\right),
\end{aligned}
$$

which is a contradiction so that (45) must be true. We thus have that $\left\|v_{2^{-k}}\right\|_{C_{\tau} H^{\gamma}\left(\langle x\rangle^{\delta}\right)}$ is uniformly bounded almost surely. Reinserting this in lemma 4.3 shows that $v_{2^{-k}}$ is (almost surely) a Cauchy sequence and thus we can conclude almost sure convergence to some $v \in C_{\tau} H^{\gamma}\left(\langle x\rangle^{\delta}\right)$. All the terms on the right hand side of (29) are bounded and convergent in $C_{\tau} H^{\gamma-2}\left(\langle x\rangle^{\delta^{\prime}}\right)$ for $\gamma \in(1,2)$ and $\delta^{\prime} \in(0, \delta)$ due to lemma 2.1, (22) and (23) so that the limit $v \in C_{\tau} H^{\gamma}\left(\langle x\rangle^{\delta}\right)$ solves (27): 


$$
\imath \partial_{t} v=\Delta v+v\left(: \nabla Y^{2}:-\varphi * \xi\right)-2 \nabla v \nabla Y+\lambda|v|^{2 \sigma} v \mathrm{e}^{-2 \sigma Y}, v(0)=v_{0}
$$

where the identity should be read (pathwise) in $C_{\tau} H^{\gamma-2}\left(\langle x\rangle^{\delta^{\prime}}\right)$. To see uniqueness, suppose we are given two solutions $\left.v, v^{\prime} \in C_{\tau} H^{\gamma}\left(\langle x\rangle^{\delta}\right)\right)$. One writes the equation for $h:=v-v^{\prime}$, deduce that $\partial_{t} h \mathrm{e}^{-Y} \in C_{T} H^{\gamma-2}\left(\langle x\rangle^{\delta}\right)$. So that the following equality is easily justified:

$$
\begin{aligned}
\frac{\mathrm{d}}{\mathrm{d} t}\left(\left\|h(\cdot) \mathrm{e}^{-Y}\right\|_{L^{2}\left(\mathbb{R}^{d}\right)}^{2}\right)(t) & =\lambda \operatorname{Im}\left(\int_{\mathbb{R}^{2}}\left(|v(t, x)|^{2 \sigma} v(t, x)-\left|v^{\prime}(t, x)\right|^{2 \sigma} v^{\prime}(t, x)\right) \bar{h} \mathrm{e}^{-(2 \sigma+2) Y(x)}\right) \\
& \leqslant K_{v, v^{\prime}}\left\|h(t) \mathrm{e}^{-Y}\right\|_{L^{2}\left(\mathbb{R}^{d}\right)}^{2},
\end{aligned}
$$

where $K_{v, v^{\prime}}$ denotes some random 'constant' which depends on $\|v\|_{C_{\tau} H^{\gamma}\left(\langle x\rangle^{\delta}\right)}$ and $\left\|v^{\prime}\right\| \|_{C_{\tau} H^{\gamma}\left(\langle x\rangle^{\delta}\right)}$ and where we applied once more $\left.|| v\right|^{2 \sigma} v-\left|v^{\prime}\right|^{2 \sigma} v^{\prime}\left|\leqslant C\left(|v|^{2 \sigma}+\left|v^{\prime}\right|^{2 \sigma}\right)\right| h \mid$ from [Caz03, p 86] together with $\left\|v(t) \mathrm{e}^{-Y}\right\|_{L^{\infty}\left(\mathbb{R}^{d}\right)} \leqslant C\|v(t)\|_{H^{\gamma}\left(\langle x\rangle^{\delta}\right)}$ (and similar for $v^{\prime}$ ) due to the embedding $H^{\gamma} \subseteq L^{\infty}$. Uniqueness then follows with Gronwall's inequality. The convergence in probablity follows similar as in [DW18] by considering first $\left\|v_{\varepsilon}-v_{2^{-k}}\right\|_{C_{\tau} H^{\gamma}\left(\langle x\rangle^{\delta}\right)}$ : redoing then the proof of lemma 4.3 but bounding $\left\|v_{2-k}\right\|_{H^{3 / 2}\left(\langle x\rangle^{\delta}\right)},\left\|v_{2^{-k}} \mathrm{e}^{-Y_{2^{-k}}}\right\|_{L^{\infty}}$ directly instead of applying lemma 4.1 we can let $k \rightarrow \infty$ and the resulting estimate yields the convergence in probability.

\section{Global existence for $\sigma<1 / 2$}

In the case $\sigma<1 / 2$ we now prove that there is a global solution to (27). As remarked at the beginning of section 4 we cannot simply use the Brezis-Gallouet inequality as we have no uniform $H^{1}$ bound yet available that controls the decay of the solution $v^{\varepsilon}$ to (29). However, we can reshape the inequality to the following statement.

Lemma 5.1. For the solution $v_{\varepsilon}$ of (29) on $[0, T]$ with $T>0$ and $\sigma<1$ we have for any $\gamma \in(1,2), \kappa>0$ and $\delta<(\gamma-1) \delta_{0}$

$\left\|v_{\varepsilon}(t) \mathrm{e}^{-Y_{\varepsilon}}\right\|_{L^{\infty}} \leqslant K_{\varepsilon}+\left(1+|\log \varepsilon|^{1+\kappa}\right)\left(1+\left\|v_{0}\right\|_{H^{1}\left(\langle x\rangle^{\left.\delta_{0}\right)}\right.}^{a}\right)+\log \left(1+\left\|v_{\varepsilon}(t)\right\|_{H^{\gamma}\left(\langle x\rangle^{-\delta}\right)}\right)$,

for $t \in[0, T]$ and some deterministic a $>0$. The sequence $\left(K_{2^{-k}}\right)_{k \in \mathbb{N}}$ one obtains for dyadic $\varepsilon$ is bounded almost surely.

Proof. Choose $\delta_{0}^{\prime} \in\left(0, \delta_{0}\right)$ large enough and $\gamma^{\prime}, \gamma^{\prime \prime} \in(1, \gamma), \gamma^{\prime \prime}<\gamma^{\prime}$, small enough such that we have $\delta<\frac{\gamma-\gamma^{\prime}}{\gamma^{\prime}} \delta_{0}^{\prime}$ and thus $\delta^{\prime}:=\frac{\gamma-\gamma^{\prime}}{\gamma} \delta_{0}^{\prime}-\frac{\gamma^{\prime}}{\gamma} \delta \in\left(0, \delta_{0}\right)$. Applying then the BrezisGallouet inequality from [Oza95] we obtain with the Besov multiplication rule ( (ii) of lemmas 2.1 and 2.7)

$$
\begin{aligned}
\left\|v_{\varepsilon}(t) \mathrm{e}^{-Y_{\varepsilon}}\right\|_{L^{\infty}} & \leqslant C\left(1+\left\|v_{\varepsilon}(t) \mathrm{e}^{-Y_{\varepsilon}}\right\|_{H^{1}}\right) \sqrt{1+\log \left(1+\left\|v_{\varepsilon}(t) \mathrm{e}^{-Y_{\varepsilon}}\right\|_{\mathcal{C}^{\gamma^{\prime \prime}-1}}\right)} \\
& \leqslant C\left(1+\left\|v_{\varepsilon}(t) \mathrm{e}^{-Y_{\varepsilon}}\right\|_{H^{1}}\right) \sqrt{1+\log \left(1+K_{\varepsilon}\left\|v_{\varepsilon}(t)\right\|_{\mathcal{C}^{\gamma^{\prime \prime}-1}\left(\langle x\rangle^{\delta^{\prime}}\right)}\right)} \\
& \leqslant K_{\varepsilon}\left(1+\left\|v_{\varepsilon}(t) \mathrm{e}^{-Y_{\varepsilon}}\right\|_{H^{1}}\right) \sqrt{1+\log \left(1+\left\|v_{\varepsilon}(t)\right\|_{\mathcal{C}^{\gamma^{\prime \prime}}\left(\langle x\rangle^{\delta^{\prime}}\right)}\right)}
\end{aligned}
$$

where we used in the last step that due to $\log K_{\varepsilon} \leqslant K_{\varepsilon}$ :

$$
\begin{aligned}
& \log \left(1+K_{\varepsilon}\left\|v_{\varepsilon}(t)\right\|_{\mathcal{C}^{\gamma^{\prime \prime}-1}\left(\langle x\rangle^{\delta^{\prime}}\right)}\right) \leqslant \log \left(K_{\varepsilon}\left(1+\left\|v_{\varepsilon}(t)\right\|_{\mathcal{C}^{\gamma^{\prime \prime}-1}\left(\langle x\rangle^{\delta^{\prime}}\right)}\right)\right) \\
& \leqslant K_{\varepsilon}+\log \left(\left(1+\left\|v_{\varepsilon}(t)\right\|_{\mathcal{C}^{\gamma^{\prime \prime}-1}\left(\langle x\rangle^{\delta^{\prime}}\right)}\right)\right) \leqslant K_{\varepsilon}\left(1+\log \left(1+\left\|v_{\varepsilon}(t)\right\|_{\mathcal{C}^{\gamma^{\prime \prime}-1}\left(\langle x\rangle^{\delta^{\prime}}\right)}\right)\right) .
\end{aligned}
$$


Application of (13) (with $\Theta=1 / 2, \zeta=1$ ) to (48) (and dropping $C_{\Theta}=C_{\frac{1}{2}}=\frac{1}{4} \leqslant 1$ ) and Besov interpolation (lemma 2.2) yields

$$
\begin{aligned}
\left\|v_{\varepsilon}(t) \mathrm{e}^{-Y_{\varepsilon}}\right\|_{L^{\infty}} \stackrel{(13)}{\leqslant} K_{\varepsilon}+\left\|v_{\varepsilon}(t) \mathrm{e}^{-Y_{\varepsilon}}\right\|_{H^{1}}^{2}+\log \left(1+\left\|v_{\varepsilon}(t)\right\|_{H^{\gamma^{\prime}}\left(\langle x\rangle^{\delta^{\prime}}\right)}\right) \\
\stackrel{\text { lemma 2.2 }}{\leqslant} K_{\varepsilon}+\left\|v_{\varepsilon}(t) \mathrm{e}^{-Y_{\varepsilon}}\right\|_{H^{1}}^{2}+\log \left(1+\left\|v_{\varepsilon}(t)\right\|_{H^{\gamma}(\langle x\rangle-\delta)}^{\frac{\gamma^{\prime}}{\gamma}} \|\left. v_{\varepsilon}(t)\right|_{L^{2}\left(\langle x\rangle^{\delta_{0}^{\prime}}\right)} ^{\frac{\gamma-\gamma^{\prime}}{\gamma}}\right) .
\end{aligned}
$$

The last term of (49) can be bounded using lemma 3.1 (together with proposition 3.2)

$$
\begin{aligned}
& \log \left(1+\left\|v_{\varepsilon}(t)\right\|_{H^{\gamma}(\langle x\rangle-\delta)}^{\frac{\gamma^{\prime}}{\gamma}}\left\|v_{\varepsilon}(t)\right\|_{L^{2}\left(\langle x\rangle^{\delta_{0}^{\prime}}\right)}^{\frac{\gamma-\gamma^{\prime}}{\gamma}}\right) \\
& \leqslant \log \left(C\left(1+\left\|v_{\varepsilon}(t)\right\|_{H^{\gamma}(\langle x\rangle-\delta)}\right)^{\frac{\gamma^{\prime}}{\gamma}}\left(1+\left\|v_{\varepsilon}(t)\right\|_{L^{2}\left(\langle x\rangle^{\delta_{0}^{\prime}}\right)}\right)^{\frac{\gamma-\gamma^{\prime}}{\gamma}}\right) \\
& \leqslant C+\frac{\gamma^{\prime}}{\gamma} \log \left(1+\left\|v_{\varepsilon}(t)\right\|_{H^{\gamma}\left(\langle x\rangle^{-\delta}\right)}\right)+\frac{\gamma-\gamma^{\prime}}{\gamma} \log \left(1+\left\|v_{\varepsilon}(t)\right\|_{L^{2}\left(\langle x\rangle^{\delta_{0}^{\prime}}\right)}\right) \\
& \leqslant K_{\varepsilon}+\log \left(1+\left\|v_{\varepsilon}(t)\right\|_{H^{\gamma}\left(\langle x\rangle^{-\delta}\right)}\right)+\left(1+\left\|v_{0}\right\|_{H^{1}\left(\langle x\rangle^{\delta_{0}}\right)}^{a}\right)
\end{aligned}
$$

where we dropped $\frac{\gamma^{\prime}}{\gamma}, \frac{\gamma-\gamma^{\prime}}{\gamma} \leqslant 1$ and used $\log x \leqslant x$ (for $x \geqslant 0$ ) in the last line. It remains to handle the term $\left\|v_{\varepsilon}(t) \mathrm{e}^{-Y_{\varepsilon}}\right\|_{H^{1}}^{2}$ in (49). By the product rule we have

$$
\left\|v_{\varepsilon}(t) \mathrm{e}^{-Y_{\varepsilon}}\right\|_{H^{1}} \stackrel{(11)}{\leqslant} C\left(\left\|v_{\varepsilon}(t) \mathrm{e}^{-Y_{\varepsilon}}\right\|_{L^{2}}+\left\|\nabla v_{\varepsilon}(t) \mathrm{e}^{-Y_{\varepsilon}}\right\|_{L^{2}}+\left\|v_{\varepsilon}(t) \mathrm{e}^{-Y_{\varepsilon}} \nabla Y_{\varepsilon}\right\|_{L^{2}}\right) .
$$

While the first term is bounded by conservation of mass, the second one can be bounded by conservation of energy: use proposition 3.2 and Sobolev embedding to estimate the right hand side of (33). For the last term we apply Hölder's inequality and lemma 2.10:

$$
\begin{aligned}
\left\|v_{\varepsilon}(t) \mathrm{e}^{-Y_{\varepsilon}} \nabla Y_{\varepsilon}\right\|_{L^{2}} & \leqslant\left\|v_{\varepsilon}(t) \mathrm{e}^{-Y_{\varepsilon}}\right\|_{L^{q}\left(\langle x\rangle^{\tilde{\delta}}\right)}\left\|\nabla Y_{\varepsilon}\right\|_{L^{q^{\prime}}(\langle x\rangle-\tilde{\delta})} \\
& \leqslant K_{\varepsilon}\left(1+\left\|v_{0}\right\|_{H^{1}\left(\langle x\rangle^{\left.\delta_{0}\right)}\right.}^{a}\right)|\log \varepsilon|^{1 / 2+\kappa / 4} \\
& \stackrel{(13)}{\leqslant} K_{\varepsilon}+\left(1+\left\|v_{0}\right\|_{H^{1}\left(\langle x\rangle^{\left.\delta_{0}\right)}\right.}^{a}\right)|\log \varepsilon|^{1 / 2+\kappa / 2},
\end{aligned}
$$

for some small $\tilde{\delta}>0$ and $\frac{1}{2}=\frac{1}{q}+\frac{1}{q^{\prime}}$ with $q^{\prime} \in(2, \infty)$ large enough so that $\tilde{\delta} q^{\prime}>2$ and where we used lemma 2.7, Sobolev embedding and corollary 3.3 in the second step. Note that we introduced an additional factor $|\log \varepsilon|^{\kappa / 4}$ from the first to the second line so that the constants $K_{\varepsilon}$ are bounded almost surely for dyadic $\varepsilon=2^{-k}$ by (22), (23) and Borel-Cantelli. Inserting (50) above shows the desired inequality.

The combination of lemma 5.1 with lemma 4.1 and 4.3 gives global existence provided $\sigma<\frac{1}{2}$.

Theorem 5.2. Let $\lambda=0$ or $\sigma \in(0,1 / 2)$ and let $v_{0}$ be given with $v_{0} \in H^{2}\left(\langle x\rangle^{\delta_{0}}\right)$ a.s. for $\delta_{0} \in\left(0, \frac{1}{2}\right)$. Consider the problem

$$
\imath \partial_{t} v=\Delta v+v\left(: \nabla Y^{2}:-\varphi * \xi\right)-2 \nabla v \nabla Y+\lambda|v|^{2 \sigma} v \mathrm{e}^{-2 \sigma Y}, v(0)=v_{0}
$$

For every $T>0$ there is a.s. a unique solution $v \in C_{T} H^{\gamma}\left(\langle x\rangle^{\delta}\right)$ on $[0, T]$ for any $\gamma \in(1,2)$ and $\delta<\left(1-\frac{\gamma}{2}\right) \delta_{0}$. The solutions $v_{\varepsilon}$ of (29) converge in probability to $v$ in $C_{T} H^{\gamma}\left(\langle x\rangle^{\delta}\right)$. 
Proof. We assume $\sigma \in(0,1 / 2)$, since the linear case $\lambda=0$ is trivially included in this range. Choose $\delta_{1} \in\left(0, \delta_{0}\right)$ large enough such that $\delta<\left(1-\frac{\gamma}{2}\right) \delta_{1}$ and choose $\delta_{2}>0$ such that $\delta=\left(1-\frac{\gamma}{2}\right) \delta_{1}+\frac{\gamma}{2}\left(-\delta_{2}\right)$. We can then interpolate between the $L^{2}\left(\langle x\rangle^{\delta_{1}}\right)$ bound from lemma 3.1 (combined with proposition 3.2) and the $H^{2}\left(\langle x\rangle^{-\delta_{2}}\right)$ bound from lemma 4.1

$$
\begin{aligned}
& \left\|v_{2-k}\right\|_{C_{T} H^{\gamma}\left(\langle x\rangle^{\delta}\right)} \stackrel{\text { lemma } 2.2}{\leqslant C}\left\|v_{2-k}\right\|_{C_{T} L^{2}\left(\langle x\rangle^{\delta_{1}}\right)}^{1-\frac{\gamma}{2}} \cdot\left\|v_{2-k}\right\|_{C_{T} H^{2}\left(\langle x\rangle^{-\delta_{2}}\right.}^{\frac{\gamma}{2}}
\end{aligned}
$$

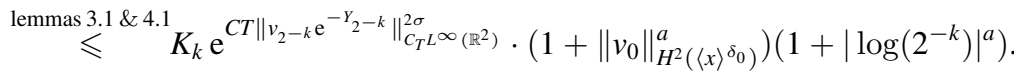

Inserting the inequality given in lemma 5.1 in the exponent on the right hand side provides us with the estimate

$$
\begin{aligned}
& \left\|v_{2-k}\right\|_{C_{T} H^{\gamma}\left(\langle x\rangle^{\delta}\right)} \leqslant \mathrm{e}^{K_{k}} \mathrm{e}^{C T\left(1+\left\|v_{0}\right\|_{H^{2}\left(\langle x\rangle^{\delta}\right.}^{a} \delta_{0}\right)\left(1+\left|\log 2^{-k}\right|\right)^{2 \sigma(1+\kappa)}} \\
& \cdot\left(1+\left\|v_{0}\right\|_{H^{2}\left(\langle x\rangle^{\delta_{0}}\right)}^{a}\right)\left(1+\left|\log \left(2^{-k}\right)\right|^{a}\right) \mathrm{e}^{\log \left(1+\left\|v_{2-k}\right\|_{T^{H} H^{\gamma}(\langle x\rangle}\right)^{2 \sigma}} \\
& \leqslant \mathrm{e}^{K_{k}} \mathrm{e}^{C T\left(1+\left\|v_{0}\right\|_{H^{2}(\langle x\rangle}^{a} \delta_{0}\right)\left(1+\left|\log 2^{-k}\right|\right)^{2 \sigma(1+\kappa)}} \cdot \mathrm{e}^{\left.\log \left(1+\left\|v_{2}-k\right\|_{T H^{\gamma}(\langle x\rangle}\right)^{2}\right)^{2 \sigma}}
\end{aligned}
$$

where we used $K_{k} \leqslant \mathrm{e}^{K_{k}}$ and in the second step that

$$
\left.\left(1+\left\|v_{0}\right\|_{H^{2}\left(\langle x\rangle^{\delta_{0}}\right)}^{a}\right)\left(1+\left|\log \left(2^{-k}\right)\right|^{a}\right) \leqslant \mathrm{e}^{\left.C T\left(1+\left\|v_{0}\right\|_{H^{2}(\langle x\rangle}^{a}\right\rangle_{0}\right)}\right)\left(1+\left|\log 2^{-k}\right|\right)^{2 \sigma(1+\kappa)} .
$$

We choose $\kappa>0$ small enough such that $2 \sigma(1+\kappa)<1$. Note that for $s \in(0,1)$ and any $\kappa^{\prime}>0$ it holds $\mathrm{e}^{\log (1+x)^{s}} \leqslant C x^{\kappa^{\prime}}$, so that we can reformulate this estimate via (13) (with $\left.\zeta=\frac{1}{2}, x=\left\|v_{2^{-k}}\right\|_{H^{\gamma}\left(\langle x\rangle^{\delta}\right)}, \Theta=\frac{1}{2}\right)$

$$
\begin{aligned}
\left\|v_{2-k}\right\|_{C_{T} H^{\gamma}\left(\left\langle\langle\rangle^{\delta}\right)\right.} & \leqslant \mathrm{e}^{K_{k}} \mathrm{e}^{C T\left(1+\left\|v_{0}\right\|_{H^{2}\left(\langle x\rangle \delta_{0}\right)}^{a}\right)\left(1+\left|\log 2^{-k}\right|\right)^{2 \sigma(1+\kappa)}}\left\|v_{2^{-k}}\right\|_{C_{T} H^{\gamma}\left(\langle x\rangle^{\delta}\right)}^{\frac{1}{2}} \\
& \stackrel{(13)}{\leqslant} \mathrm{e}^{K_{k}} \mathrm{e}^{C T\left(1+\left\|v_{0}\right\|_{H^{2}\left(\langle x\rangle^{\delta}\right)}^{a}\right)\left(1+\left|\log 2^{-k}\right|\right)^{2 \sigma(1+\kappa)}}+\frac{1}{2}\left\|v_{2^{-k}}\right\|_{C_{T} H^{\gamma}\left(\langle x\rangle^{\delta}\right) .}
\end{aligned}
$$

Subtracting the last term and multiplying both sides by 2 yields

$$
\left\|v_{2-k}\right\|_{C_{T} H^{\gamma}\left(\langle x\rangle^{\delta}\right)} \leqslant \mathrm{e}^{K_{k}} \mathrm{e}^{C T\left(1+\left\|v_{0}\right\|_{H^{2}\left(\langle x)^{\delta} \delta_{0}\right.}^{a}\right)\left(1+\left|\log 2^{-k}\right|\right)^{2 \sigma(1+\kappa)}} .
$$

Reinserting this into the Brezis-Gallouet inequality, lemma 5.1, (and using $2 \sigma \leqslant 1$ ) gives

$$
\left\|v_{2-k} \mathrm{e}^{-Y_{2-k}}\right\|_{C_{T} L^{\infty}\left(\mathbb{R}^{2}\right)} \leqslant K_{k}+\left(1+\left|\log 2^{-k}\right|^{1+\kappa}\right)\left(1+\left\|v_{0}\right\|_{H^{2}\left(\langle x\rangle^{\delta_{0}}\right)}^{a}\right) .
$$

Recall that $\kappa>0$ was chosen such that such that $s:=2 \sigma(1+\kappa)<1$. Combining this estimate with lemma 4.3 we end up with

$\left\|v_{2-k}-v_{2-k-1}\right\|_{C_{T} H^{\gamma}\left(\langle x\rangle^{\delta}\right)} \leqslant 2^{-k \kappa^{\prime}} \mathrm{e}^{K_{k}} \mathrm{e}^{C T\left(1+\left\|v_{0}\right\|_{H^{2}\left(\langle x)^{\delta}\right)}^{a}\right)\left(1+\left|\log 2^{-k}\right|\right)^{s}} \leqslant C\left(v_{0}\right) \mathrm{e}^{K_{k} 2^{-k \kappa^{\prime} / 2}}$

for $\kappa^{\prime}>0, C\left(v_{0}\right)>0$ and where we used $s<1$ in the second step and once more $\mathrm{e}^{\log (1+x)^{s}} \leqslant C x^{\kappa^{\prime}}$. We therefore conclude that $v_{2^{-k}}$ is a Cauchy sequence in $C_{T} H^{\gamma}\left(\langle x\rangle^{\delta}\right)$ and the rest of the proof follows as in theorem 4.4. 


\section{Appendix. Proof of proposition 2.11}

We first treat the case $\sigma<1$. We prove existence for equation (28) and then translate the result to the transformed equation. As above, we fix the randomness and work a.s. Let $N \in \mathbb{N}$ and consider the equation:

$$
\imath \partial_{t} u_{\varepsilon, N}=\Delta u_{\varepsilon, N}+u_{\varepsilon, N}\left(\xi_{\varepsilon, N}-c_{\varepsilon, N}\right)+\lambda\left|u_{\varepsilon, N}\right|^{2 \sigma} u_{\varepsilon, N}, u_{\varepsilon, N}(0)=\mathrm{e}^{-Y_{\varepsilon, N}} v_{0},
$$

where $\xi_{\varepsilon, N}=\theta\left(\frac{\xi_{\varepsilon}}{N}\right) \xi_{\varepsilon}$ and $Y_{\varepsilon, N}=G * \xi_{\varepsilon, N}$ for some smooth positive function $\theta$ equal to 1 on $[-1,1]$ and to 0 outside $[-2,2]$. This equation contains only bounded and smooth terms, it is classical that it has a unique and global solution in $C\left(\mathbb{R}^{+}, H^{1}\right)$ a.s. (see [Caz03]) and, since the initial data is in $H^{2}$, it is in $C\left(\mathbb{R}^{+}, H^{2}\right)$ a.s. (see [Kat87]). The following estimate, similar to lemma 3.1, can be justified rigorously by the same argument as in [Caz03], lemma 6.5.2. Let us write:

$$
\begin{aligned}
& \frac{\mathrm{d}}{\mathrm{d} t}\left(\int_{\mathbb{R}^{2}}\left|\langle x\rangle^{\delta} u_{\varepsilon, N}(\cdot, x)\right|^{2} \mathrm{~d} x\right)(t)=2 \operatorname{Re}\left(\int_{\mathbb{R}^{2}}\langle x\rangle^{2 \delta} \partial_{t} u_{\varepsilon, N}(t, x) \cdot \bar{u}_{\varepsilon, N}(t, x) \mathrm{d} x\right) \\
& =2 \operatorname{Im}\left(\int_{\mathbb{R}^{2}}\langle x\rangle^{2 \delta} \Delta u_{\varepsilon, N}(t, x) \bar{u}_{\varepsilon, N}(t, x) \mathrm{d} x\right)=-2 \operatorname{Im}\left(\int_{\mathbb{R}^{2}} \nabla\langle x\rangle^{2 \delta} \cdot \nabla u_{\varepsilon, N}(t, x) \bar{u}_{\varepsilon, N}(t, x) \mathrm{d} x\right) \\
& \leqslant C_{\delta} \int_{\mathbb{R}^{2}}\langle x\rangle^{2 \delta-1}\left|\nabla u_{\varepsilon, N}\right|\left|u_{\varepsilon, N}(t, x)\right| \mathrm{d} x \\
& \leqslant C_{\delta}\left(\int_{\mathbb{R}^{2}}\left|\nabla u_{\varepsilon, N}(\cdot, x)\right|^{2} \mathrm{~d} x\right)^{1 / 2}\left(\int_{\mathbb{R}^{2}}\left|\langle x\rangle^{\delta} u_{\varepsilon, N}(\cdot, x)\right|^{2} \mathrm{~d} x\right)^{1 / 2} \\
& \leqslant C_{\delta} \int_{\mathbb{R}^{2}}\left|\nabla u_{\varepsilon, N}(\cdot, x)\right|^{2} \mathrm{~d} x+\int_{\mathbb{R}^{2}}\left|\langle x\rangle^{\delta} u_{\varepsilon, N}(\cdot, x)\right|^{2} \mathrm{~d} x .
\end{aligned}
$$

We deduce from Gronwall lemma and $u_{\varepsilon, N} \in C\left(\mathbb{R}^{+}, H^{1}\right)$ that $u_{\varepsilon, N} \in C\left(\mathbb{R}^{+}, L^{2}\left(\langle x\rangle^{\delta_{0}}\right)\right)$. Also, it follows from the above reference that the energy and mass are conserved:

$$
\begin{aligned}
N\left(u_{\varepsilon, N}(t)\right) & =\int_{\mathbb{R}^{2}}\left|u_{\varepsilon, N}(t, x)\right|^{2} \mathrm{~d} x=N\left(u_{\varepsilon, N}(0)\right), \\
H\left(u_{\varepsilon, N}(t)\right) & =\int_{\mathbb{R}^{2}}\left(\frac{1}{2}\left|\nabla u_{\varepsilon, N}(t, x)\right|^{2}-\frac{1}{2}\left|u_{\varepsilon, N}(t, x)\right|^{2} \xi_{\varepsilon, N}-\frac{\lambda}{2 \sigma+2}\left|u_{\varepsilon, N}(t, x)\right|^{2 \sigma+2}\right) \mathrm{d} x \\
& =H\left(u_{\varepsilon, N}(0)\right) .
\end{aligned}
$$

We use similar, and simpler, arguments as in proposition 3.2. By Sobolev embedding, (ii) in lemma 2.1, interpolation, lemma 2.2, and (13) we have:

$$
\begin{aligned}
\left.\left|\int_{\mathbb{R}^{2}} \frac{\lambda}{2 \sigma+2}\right| u_{\varepsilon, N}(t, x)\right|^{2 \sigma+2} \mathrm{~d} x \mid & \leqslant C_{\sigma}\left\|u_{\varepsilon, N}(t)\right\|_{L^{2}}^{2}\left\|u_{\varepsilon, N}(t)\right\|_{H^{1}}^{2 \sigma} \\
& \leqslant C_{\sigma}\left(\left\|u_{\varepsilon, N}(t)\right\|_{L^{2}}^{2 \sigma+2}+\left\|u_{\varepsilon, N}(t)\right\|_{L^{2}}^{2}\left\|\nabla u_{\varepsilon, N}(t)\right\|_{L^{2}}^{2 \sigma}\right) \\
& \leqslant \frac{1}{4}\left\|\nabla u_{\varepsilon, N}(t)\right\|_{L^{2}}^{2}+C_{\sigma, \lambda}\left(\left\|u_{\varepsilon, N}(t)\right\|_{L^{2}}^{2 /(1-\sigma)}+\left\|u_{\varepsilon, N}(t)\right\|_{L^{2}}^{2 \sigma+2}\right) \\
& \leqslant \frac{1}{4}\left\|\nabla u_{\varepsilon, N}(t)\right\|_{L^{2}}^{2}+C_{\sigma, \lambda}\left(\left\|u_{\varepsilon, N}(t)\right\|_{L^{2}}^{2 /(1-\sigma)}+1\right) .
\end{aligned}
$$

Moreover, $\xi_{\varepsilon, N}$ is clearly bounded uniformly w.r.t. $N$-but not w.r.t $\varepsilon$-in $L^{\infty}\left(\langle x\rangle^{-\delta_{0}}\right)$ and we have:

$$
\left.\left|\int_{\mathbb{R}^{2}}\right| u_{\varepsilon, N}(t, x)\right|^{2} \xi_{\varepsilon, N} \mid \mathrm{d} x \leqslant C_{\varepsilon, \delta_{0}}\left\|u_{\varepsilon, N}(t)\right\|_{L^{2}\left(\langle x\rangle^{\delta_{0}}\right)}^{2}
$$


Inserting (A.5), (A.6) in (A.4) and using (A.3) gives:

$\left\|\nabla u_{\varepsilon, N}(t)\right\|_{L^{2}}^{2} \leqslant C_{\sigma, \lambda, \varepsilon, \delta_{0}}\left(H\left(u_{\varepsilon, N}(0)\right)+\left\|u_{\varepsilon, N}(0)\right\|_{L^{2}}^{2 /(1-\sigma)}+1+\left\|u_{\varepsilon, N}(t)\right\|_{L^{2}\left(\langle x\rangle \delta_{0}\right)}^{2}\right)$

and from (A.2) with Gronwall lemma, for $T \geqslant 0$,

$\sup _{t \in[0, T]}\left\|u_{\varepsilon, N}(t)\right\|_{L^{2}\left(\langle x\rangle^{\delta_{0}}\right)}^{2} \leqslant C_{\sigma, \lambda, \varepsilon, \delta_{0}, T}\left(H\left(u_{\varepsilon, N}(0)\right)+\left\|u_{\varepsilon, N}(0)\right\|_{L^{2}}^{2 /(1-\sigma)}+\mid u_{\varepsilon, N}(0) \|_{L^{2}\left(\langle x\rangle^{\delta_{0}}\right)}^{2}+1\right)$.

With (A.3) and (A.7), this gives the bound:

$\sup _{t \in[0, T]}\left\|u_{\varepsilon, N}(t)\right\|_{H^{1}}^{2} \leqslant C_{\sigma, \lambda, \varepsilon, \delta_{0}, T}\left(H\left(u_{\varepsilon, N}(0)\right)+\left\|u_{\varepsilon, N}(0)\right\|_{L^{2}}^{2 /(1-\sigma)}+\mid u_{\varepsilon, N}(0) \|_{L^{2}\left(\langle x\rangle^{\delta_{0}}\right)}^{2}+1\right)$.

We then obtain a $H^{2}$ bound thanks to Brezis-Gallouet inequality [BG80]. We set $w_{\varepsilon, N}=\partial_{t} u_{\varepsilon, N}$ and obtain as in lemma 4.1:

$\frac{\mathrm{d}}{\mathrm{d} t}\left\|w_{\varepsilon, N}\right\|_{L^{2}}^{2} \leqslant 2 \sigma|\lambda|\left\|u_{\varepsilon, N}\right\|_{L^{\infty}}^{2 \sigma}\left\|w_{\varepsilon, N}\right\|_{L^{2}}^{2} \leqslant C_{\sigma, \lambda}\left\|u_{\varepsilon, N}\right\|_{H^{1}}^{2 \sigma}\left(1+\log \left(1+\left\|u_{\varepsilon, N}\right\|_{H^{2}}\right)\right)^{\sigma}\left\|w_{\varepsilon, N}\right\|_{L^{2}}^{2}$.

We then have, using the equation:

$$
\begin{aligned}
\left\|u_{\varepsilon, N}\right\|_{H^{2}} & \leqslant C\left(\left\|\Delta u_{\varepsilon, N}\right\|_{L^{2}}+\left\|u_{\varepsilon, N}\right\|_{H^{1}}\right) \\
& \leqslant C_{\lambda}\left(\left\|w_{\varepsilon, N}\right\|_{H^{2}}+\left\|u_{\varepsilon, N}\right\|_{L^{2 \sigma+2}}^{2 \sigma+2}+\left\|\xi_{\varepsilon, N} u_{\varepsilon, N}\right\|_{L^{2}}+\left\|u_{\varepsilon, N}\right\|_{H^{1}}\right) \\
& \leqslant C_{\lambda}\left(\left\|w_{\varepsilon, N}\right\|_{H^{2}}+\left\|u_{\varepsilon, N}\right\|_{H^{1}}^{2 \sigma+2}+1+\left\|u_{\varepsilon, N}\right\|_{L^{2}\left(\left\langle x \delta^{\delta_{0}}\right)\right.}\right) .
\end{aligned}
$$

It is then lengthy but not difficult to deduce a bound on $\sup _{t \in[0, T]}\left\|w_{\varepsilon, N}\right\|_{L^{2}}^{2}$, and therefore on $\sup _{t \in[0, T]}\left\|u_{\varepsilon, N}\right\|_{H^{2}}^{2}$, independent of $N$ :

$$
\sup _{t \in[0, T]}\left\|u_{\varepsilon, N}\right\|_{H^{2}}^{2} \leqslant C\left(\sigma, \lambda, \varepsilon, \delta_{0}, T,\left\|u_{\varepsilon, N}(0)\right\|_{H^{2}},\left\|u_{\varepsilon, N}(0)\right\|_{L^{2}\left(\langle x\rangle \delta_{0}\right)}\right) .
$$

A bound on $\sup _{t \in[0, T]}\left\|\partial_{t} u_{\varepsilon, N}\right\|_{L^{2}}$ follows using the equation. Thus we have proved that $\left(u_{\varepsilon, N}\right)_{N \in \mathbb{N}}$ is bounded in $\left.C\left([0, T] ; H^{2} \cap L^{2}\left(\langle x\rangle^{\delta_{0}}\right)\right) \cap C^{1}\left([0, T] ; L^{2}\right)\right)$. By Ascoli theorem, we obtain a convergent subsequence $\left(u_{\varepsilon, N_{k}}\right)_{k \in \mathbb{N}}$ in $C\left([0, T], L^{2}\left(\langle x\rangle^{\delta}\right)\right)$ for $\delta<\delta_{0}$ and by interpolation in $C\left([0, T], H^{\gamma}\left(\langle x\rangle^{(1-\gamma / 2) \delta}\right)\right)$. We let $k \rightarrow \infty$ and prove that the limit $u_{\varepsilon}$ satisfies equation (28). By the Sobolev embedding $H^{\gamma} \subset L^{\infty}$, there is no other solution in $C\left([0, T], H^{\gamma}\right)$ to (28) and we deduce that the whole $\left(u_{\varepsilon, N}\right)_{N \in \mathbb{N}}$ converges to $u_{\varepsilon}$.

Note that letting $N \rightarrow \infty$ in (A.3), (A.4), we prove that the mass and energy are conserved also at the limit. Moreover letting $N \rightarrow \infty$ in (A.8) gives $u^{\varepsilon} \in L^{\infty}\left(0, T ; H^{2}\right)$.

It remains to set $v_{\varepsilon}=u_{\varepsilon} \mathrm{e}^{Y_{\varepsilon}}$ to finish the proof for $\sigma<1$.

When $\lambda \leqslant 0$, we proceed differently and use another approximation step. For any $N \in \mathbb{N}$, we define $Y_{\varepsilon_{N}}=G * \xi_{\varepsilon, N}, \xi_{\varepsilon, N}=\theta_{N} \xi$ where $\theta_{N}(x)=\theta\left(\frac{x}{N}\right)$ and ${\widetilde{\nabla Y_{\varepsilon}^{2}}}^{N}:=\theta_{N}: \widetilde{\nabla Y_{\varepsilon}^{2}}:$. Since $\theta_{N}$ is smooth with all derivatives uniformly bounded in $N$, these objects satisfy the same bounds as $Y_{\varepsilon}, \xi_{\varepsilon}$ and $\overline{: \nabla Y_{\varepsilon}^{2}}:$, uniformly in $N$.

We the consider the equation:

$$
\imath \partial_{t} v_{\varepsilon, N}=\Delta v_{\varepsilon, N}+v_{\varepsilon, N}:{\widetilde{\nabla Y_{\varepsilon}^{2}}}^{N}:-2 \nabla v_{\varepsilon, N} \nabla Y_{\varepsilon, N}+\lambda\left|v_{\varepsilon, N} \mathrm{e}^{-Y_{\varepsilon, N}}\right|^{2 \sigma} v_{\varepsilon, N}, v_{\varepsilon, N}(0)=v_{0} .
$$

We do on this equations all the estimates used in lemma 3.1, proposition 3.2, corollary 3.3, lemmas 4.1, 4.3 and obtain that all the bounds stated in these results hold for $v_{\varepsilon, N}$ with bounds independent on $\varepsilon$ and on $N$. Arguing as in the proof of theorem 4.4, we deduce that $v_{\varepsilon, N}$ is 
bounded in $C_{\tau_{0}} H^{\gamma}\left(\langle x\rangle^{\delta}\right)$ for any $\gamma \in(1,2)$ and a random time $\tau_{0}$ independent on $\varepsilon$ since it is characterized by (46). Using (A.9) we obtain that $v_{\varepsilon, N}$ is bounded also in $C^{1}\left(\left[0, \tau_{0}\right] ; H^{\gamma-2}\left(\langle x\rangle^{\delta}\right)\right.$.

Arguing as above, we obtain a solution of (28) in $C_{\tau_{0}} H^{\gamma}\left(\langle x\rangle^{\delta}\right)$. As above, it is easy to see that mass and energy are conserved on $\left[0, \tau_{0}\right]$. Moreover, the estimate obtained as in lemma implies that the solution is in $L^{\infty}\left(0, \tau_{0} ; H^{2}\left(\langle x\rangle^{-\delta}\right)\right.$ for $\delta>0$.

\section{ORCID iDs}

Jörg Martin ํㅜ https://orcid.org/0000-0001-5066-7661

\section{References}

[AC15] Allez R and Chouk K 2015 The continuous Anderson hamiltonian in dimension two (in preparation)

[And58] Anderson P W 1958 Absence of diffusion in certain random lattices Phys. Rev. 109 1492-505

[BCD11] Bahouri H, Chemin J-Y and Danchin R 2011 Fourier Analysis and Nonlinear Partial Differential Equations (Berlin: Springer)

[Ber98] Bergé L 1998 Wave collapse in physics: principles and applications to light and plasma waves Phys. Rep. 303 259-370

[BG80] Brezis H and Gallouet T 1980 Nonlinear Schrödinger evolution equations Nonlinear Anal. Theory Methods Appl. 4 677-81

[Caz79] Cazenave T 1979 Equations de schrödinger non linéaires en dimension deux Proc. $R$. Soc. Edinburgh A 84 327-46

[Caz03] Cazenave T 2003 Semilinear Schrödinger Equations (Courant Lecture Notes in Mathematics) (Providence, RI: American Mathematical Society)

[CC18] Catellier R and Chouk K 2018 Paracontrolled distributions and the 3-dimensional stochastic quantization equation Ann. Probab. 46 2621-79

[Con12] Conti C 2012 Solitonization of the Anderson localization Phys. Rev. A 86061801

[DPD03] Da Prato G and Debussche A 2003 Strong solutions to the stochastic quantization equations Ann. Probab. 31 1900-16

[DW18] Debussche A and Weber H 2018 The Schrödinger equation with spatial white noise potential Electron. J. Probab. 2328

[FC10] Folli V and Conti C 2010 Frustrated brownian motion of nonlocal solitary waves Phys. Rev. Lett. 104193901

[GIP15] Gubinelli M, Imkeller P and Perkowski N 2015 Paracontrolled distributions and singular PDEs Forum Math. Pi 3 e6

[Hai14] Hairer M 2014 A theory of regularity structures Inventiones Math. 198 269-504

[HKPS93] Hida T, Kuo H-H, Potthoff J and Streit W 1993 White Noise: an Infinite Dimensional Calculus (Mathematics and its Applications) (Dordrecht: Kluwer)

[HL15] Hairer M and Labbé C 2015 A simple construction of the continuum parabolic anderson model on $\mathbf{R}^{2}$ Electron. Commun. Probab. 2011

[Jan97] Janson S 1997 Gaussian Hilbert Spaces (Cambridge Tracts in Mathematics) (Cambridge: Cambridge University Press)

[Kat87] Kato T 1987 On nonlinear Schrödinger equations Ann. Inst. Henri Poincare Phys. Theor. 46 113-29

[MP17] Martin J and Perkowski N 2017 Paracontrolled distributions on Bravais lattices and weak universality of the $2 \mathrm{~d}$ parabolic Anderson model Ann. Inst. Henri Poincaré Probabilités et Statistiques in preparation

[Nua06] Nualart D 2006 The Malliavin Calculus and Related Topics vol 1995 (Berlin: Springer)

[Oza95] Ozawa T 1995 On critical cases of sobolev's inequalities J. Funct. Anal. 127 259-69

[PT16] Prömel D J and Trabs M 2016 Rough differential equations driven by signals in besov spaces J. Differ. Equ. 260 5202-49 
[SSV14] Sickel W, Skrzypczak L and Vybíral J 2014 Complex interpolation of weighted besov and Lizorkin-Triebel spaces Acta Math. Sin. Engl. Ser. 30 1297-323

[Tri83] Triebel H 1983 Theory of Function Spaces (Basel: Birkhäuser)

[Tri06] Triebel H 2006 Theory of Function Spaces III (Basel: Birkhäuser)

[WM15] Weber H and Mourrat J-C 2017 Global well-posedness of the dynamic $\phi^{4}$ model in the plane Ann. Probab. 45 2398-476

[Yos74] Yosida K 1974 Functional Analysis (Berlin: Springer) 